\title{
On the role of artificial intelligence in medical imaging of COVID-19
}

\section{Review Article}

\section{Author(s):}

Born, Jannis; Beymer, David; Rajan, Deepta; Coy, Adam; Mukherjee, Vandana V.; Manica, Matteo; Prasanna, Prasanth; Ballah, Deddeh; Guindy, Michal; Shaham, Dorith; Shah, Pallav L.; Karteris, Emmanouil; Robertus, Jan L.; Gabrani, Maria; Rosen-Zvi, Michal

\section{Publication date:}

2021-06-11

\section{Permanent link:}

https://doi.org/10.3929/ethz-b-000490430

\section{Rights / license:}

Creative Commons Attribution 4.0 International

\section{Originally published in:}

Patterns 2(6), https://doi.org/10.1016/j.patter.2021.100269 


\section{On the role of artificial intelligence in medical imaging of COVID-19}

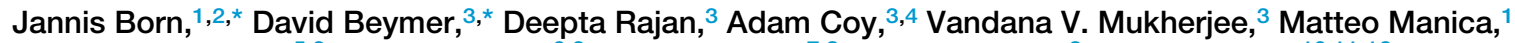
Prasanth Prasanna, ${ }^{5,3}$ Deddeh Ballah, ${ }^{6,3}$ Michal Guindy, ${ }^{7,8}$ Dorith Shaham, ${ }^{9}$ Pallav L. Shah, ${ }^{10,11,12}$ Emmanouil Karteris, ${ }^{13}$ Jan L. Robertus, ${ }^{10,12}$ Maria Gabrani, ${ }^{1}$ and Michal Rosen-Zvi ${ }^{14,15}$ ${ }^{1}$ IBM Research Europe, Zurich, Switzerland

${ }^{2}$ Department for Biosystems Science \& Engineering, ETH Zurich, Zurich, Switzerland

${ }^{3}$ IBM Almaden Research Center, San Jose, CA, USA

${ }^{4}$ Vision Radiology, Dallas, TX, USA

${ }^{5}$ Department of Radiology and Imaging Sciences, University of Utah Health Sciences Center, Salt Lake City, UT, USA

${ }^{6}$ Department of Radiology, Seton Medical Center, Daly City, CA, USA

${ }^{7}$ Assuta Medical Centres Radiology, Tel-Aviv, Israel

${ }^{8}$ Ben-Gurion University Medical School, Be'er Sheva, Israel

${ }^{9}$ Department of Radiology, Hadassah-Hebrew University Medical Center, Faculty of Medicine, Hebrew University of Jerusalem, Jerusalem, Israel

${ }^{10}$ Royal Brompton and Harefield Hospitals, Guy's and St Thomas' NHS Foundation Trust, London, UK

${ }^{11}$ Chelsea \& Westminster Hospital, London, UK

${ }^{12}$ National Heart \& Lung Institute, Imperial College London, London, UK

${ }^{13}$ College of Health, Medicine and Life Sciences, Brunel University London, London, UK

${ }^{14}$ IBM Research Haifa, Haifa, Israel

${ }^{15}$ Faculty of Medicine, The Hebrew University of Jerusalem, Jerusalem, Israel

*Correspondence: jab@zurich.ibm.com (J.B.), beymer@us.ibm.com (D.B.)

https://doi.org/10.1016/j.patter.2021.100269

THE BIGGER PICTURE During the COVID-19 pandemic, medical imaging (CT, X-ray, ultrasound) has played a key role in addressing the magnified need for speed, low cost, ubiquity, and precision in patient care. The contemporary digitization of medicine and rise of artificial intelligence (Al) induce a quantum leap in medical imaging: Al has proven equipollent to healthcare professionals across a diverse range of tasks, and hopes are high that Al can save time and cost and increase coverage by advancing rapid patient stratification and empowering clinicians.

This review bridges medical imaging and Al in the context of COVID-19 and conducts the largest systematic review of the literature in the field. We identify several gaps and evidence significant disparities between clinicians and Al experts and foresee a need for improved, interdisciplinary collaboration to develop robust Al solutions that can be deployed in clinical practice.

The key challenges on that roadmap are discussed alongside recommended solutions.

\section{SUMMARY}

Although a plethora of research articles on AI methods on COVID-19 medical imaging are published, their clinical value remains unclear. We conducted the largest systematic review of the literature addressing the utility of $\mathrm{Al}$ in imaging for COVID-19 patient care. By keyword searches on PubMed and preprint servers throughout 2020, we identified 463 manuscripts and performed a systematic meta-analysis to assess their technical merit and clinical relevance. Our analysis evidences a significant disparity between clinical and Al communities, in the focus on both imaging modalities (AI experts neglected CT and ultrasound, favoring X-ray) and performed tasks ( $71.9 \%$ of Al papers centered on diagnosis). The vast majority of manuscripts were found to be deficient regarding potential use in clinical practice, but $2.7 \%(n=12)$ publications were assigned a high maturity level and are summarized in greater detail. We provide an itemized discussion of the challenges in developing clinically relevant Al solutions with recommendations and remedies. 


\section{INTRODUCTION}

The COVID-19 pandemic has created a desperate need for fast, ubiquitous, accurate, and low-cost tests, and lung imaging is a key complementary tool in the diagnosis and management of COVID-19. ${ }^{1,2}$ According to the American College of Radiology (ACR) and the Fleischner Society Consensus Statement, imaging of COVID-19 is indicated in case of worsening respiratory symptoms, and, in a resource-constrained environment, for triage of patients with moderate to severe clinical features and a high probability of disease. ${ }^{3,4}$ This involves two main tasks. The first is diagnosis, including incidental diagnosis and providing support evidence in clinical situations in which a false-negative RT-PCR test is suspected. The second task is to help evaluate treatment outcomes, disease progression, and anticipated prognosis. The field of artificial intelligence (Al) in medical imaging (MI) is growing in the context of COVID-19, ${ }^{5-7}$ and hopes are high that $\mathrm{Al}$ can support clinicians and radiologists on these tasks. In this paper, we review the current progress in the development of $\mathrm{Al}$ technologies for $\mathrm{Ml}$ to assist in addressing the COVID-19 pandemic, discuss how Al meets the identified gaps, and share observations regarding the maturity and clinical relevancy of these developments.

\section{State of artificial intelligence in radiology}

Radiologists play a crucial role in interpreting medical images for the diagnosis and prognosis of disease. Although Al technologies have recently demonstrated performance that matches radiologists' accuracy in a number of specific tasks, it remains unclear whether radiologists who adopt Al assistance will replace those who do not. As Celi et al. put it in 2019, "the question is not whether computers can outperform human in specific tasks, but how humanity will embrace and adopt these capabilities into the practice of medicine." 8 A stepping stone toward this longterm vision, however, is the development of Al models that can compete with humans on specific tasks, and a pioneer in that progress is the tremendous success in using Al for detection of breast cancer in screening mammography ${ }^{9-12}$-a success reported by multiple research groups, achieved after 10 years of effort and crowned by OPTIMAM, a database with a total cohort of $>150,000$ clients. $^{13}$

Similarly, up to 2020 , significant progress has been made in diagnosing lung conditions using chest X-ray (CXR) and computed tomography (CT), driven by access to publicized annotated datasets. For example, deep learning (DL)-based approaches outperform radiologists in detecting several pulmonary conditions from $\mathrm{CXR}^{14}$ and malignancy of lung nodules in lowdose CT. ${ }^{15}$ Recently, technologies aiming to assist radiologists in such tasks have been made available on the market. ${ }^{16} \mathrm{Howev-}$ er, several key challenges limit the feasibility of adopting these solutions in practice, namely: (1) poor model generalization due to systemic biases; (2) lack of model interpretability; and (3) non-scalable image annotation processes. Interestingly, similar observations were revealed in the study at hand.

\section{Motivation and contributions}

The recent acceleration of publications intersecting Al and imaging for COVID-19 brings a need for rigorous comparative evaluation of papers to summarize and highlight trends to a broad clin- ical audience. Previous review papers on COVID-19 either focused on a technical assessment of $\mathrm{Al}$ in imaging ${ }^{6}$ or elaborated on the role of imaging. ${ }^{1}$ Related systematic reviews were either not devoted specifically to imaging ${ }^{17,18}$ or used extremely small sample sizes $(\mathrm{N}=11) .{ }^{19}$ In contrast, this paper attempts to bridge clinical and technical perspectives by providing a comprehensive overview to guide researchers toward working on the most pressing problems in automating lung image analysis for COVID-19. Most related to our work, Roberts et al. ${ }^{20}$ very recently conducted a systematic review of 62 studies and claim that none of the models are of potential clinical use. While the objective of our work is similar and we also find that the vast majority of manuscripts suffer from methodological flaws, we identify 12 publications that meet substantially higher standards than the remaining manuscripts. Moreover, this work is less focused on assessing individual contributions and more on extracting current trends in the field in a rigorous and comprehensive manner.

Overall, we herein provide the largest systematic meta-analysis of Al in MI of COVID-19 to date. Manually analyzing 463 publications throughout all of 2020 (Figure 1), we attempt to draw a cohesive picture on the current efforts in the field and highlight future challenges, especially related to the cooperation of clinicians and Al experts. While we focus on the lung as the primary organ of SARS-CoV-2 infection, we note the significance of extrapulmonary manifestations. ${ }^{21}$

\section{RESULTS}

\section{Progress in Al for medical imaging}

In recent years, Al solutions have shown to be capable of assisting radiologists and clinicians in detecting diseases, assessing severity, automatically localizing and quantifying disease features, or providing an automated assessment of disease prognosis. Al for MI has received extraordinary attention in 2020 , as attested by a multitude of interdisciplinary projects attempting to blend $\mathrm{Al}$ technologies with knowledge from $\mathrm{Ml}$ in order to combat COVID-19. A keyword search combining Al and MI revealed 2,563 papers in 2019, while 2020 has seen more than twice the number of such papers $(5,401$, cf. Figure 2$)$. Of these publications, 827 are related to COVID-19, indicating that COVID-19 has accelerated the development of $\mathrm{Al}$ in $\mathrm{MI}$.

\section{Lung and breast imaging comparison}

To enable a perspective on the emergence of Al for MI of COVID19 , we have compiled a comparison of the progress of automatic analysis in breast and lung imaging, as defined in the literature above, from between 2017 and 2020. Figure 3 (left) shows a stable growth of papers in $\mathrm{Al}$ on both lung and breast imaging over the years 2017-2019. In 2020, the rise of lung-related papers has been accelerated by COVID-19 with a doubling in the first half of 2020 compared with the second half of 2019 as well as a doubling of 2020 compared with 2019, whereas the trend on Al on mammography imaging remained unaltered compared with previous years.

\section{Lung imaging modality comparison}

To compare the impact of individual modalities, Figure 3 (right) shows that 2019 witnessed a stable trend of $\sim 100-120$ papers per quarter on $\mathrm{Al}$ whereas with the COVID-19 outbreak in 2020 , numbers soared to $164,352,372$, and 405 papers for 

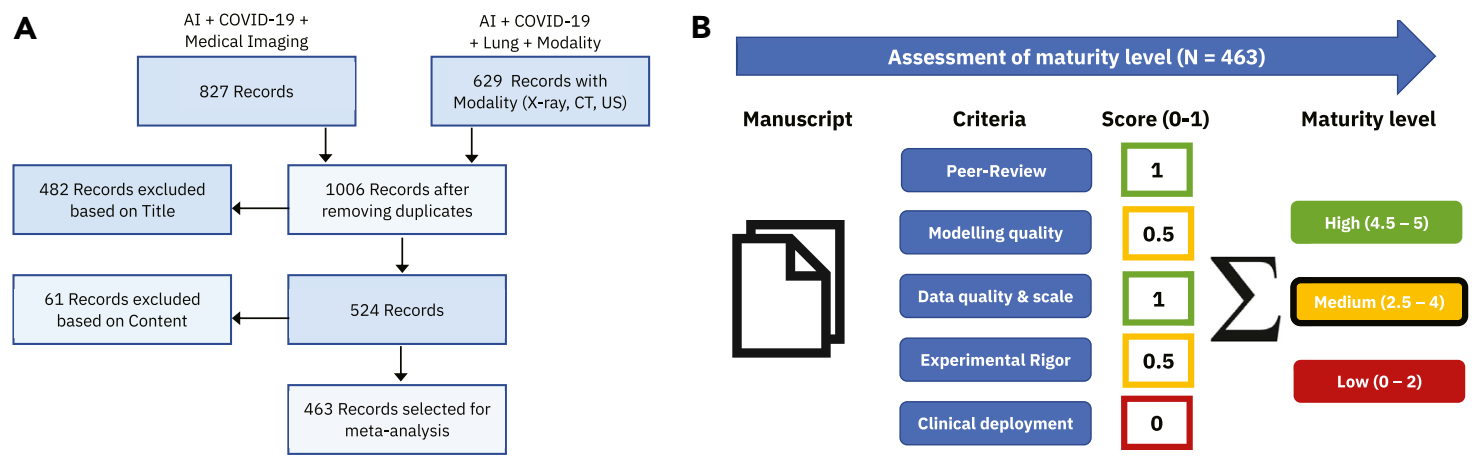

Figure 1. Overview of systematic review and meta-analysis

(A) PRISMA flowchart illustrating the study selection used in the systematic review. Publication keyword searches on PubMed, arXiv, biorXiv, and medRxiv for all of 2020 were performed using two parallel streams. After duplicate matches were removed, titles were screened manually and a selection of 463 relevant manuscripts was chosen for manual review.

(B) Flowchart for quality/maturity assessment of papers. Each manuscript received a score of between 0 and 1 for five categories. Based on the total grade, a low, medium, or high maturity level was assigned. Details on the scoring system and scores for individual papers can be found in supplemental information.

quarter 1 (Q1) to Q4 in 2020, respectively. This rise was spontaneously evoked by COVID-19, as excluding papers mentioning COVID-19 would have resulted in a continuation of the stable trend (see lightly shaded bars) of a hypothetical $\sim 120-160$ publications. Notably, the relative contributions of the modalities changed toward CXR from 2019 to 2020 (shares of $71 \%$ versus $63 \%$ for CT, 27\%-35\% for CXR, and $2 \%$ for ultrasound, respectively). Moreover, for non-COVID-19 papers, the ratio between preprints and PubMed indexed papers for $\mathrm{Al}$ in breast and chest is $29 \%$ and $37 \%$ from 2017 to 2019 , respectively; for COVID-19 related papers, this ratio rose to $58 \%$.

\section{Broad insights from meta-analysis}

By focusing on CT, CXR, and ultrasound, we quantified the publication efforts of Al for MI of COVID-19 and identified 463 papers, which were included in a manual meta-analysis to review the maturity of the Al technologies and the trends in the rapidly evolving field. The full spreadsheet with individual scores for each publication is available in supplemental information.

Disparity between clinical and AI communities

Of the 4,977 papers about MI and COVID-19 (see Figure 2, right), 2,496 are specific to modalities as shown in Figure 4 (left), indicating a dominance of CT in clinical papers (84\%), followed by CXR (10\%) and lung ultrasound (LUS) (6\%). By using publication counts as an indirect indicator of scientific response, we observe a mismatch in the focus of the Al community in comparison with the clinical community, as illustrated by the distribution of papers per modality in Figure 4 (right) that shows a clear dominance of CXR (50\%) across Al papers.

In addition, the vast majority (72\%) of papers focused on diagnosis of COVID-19 over tasks such as severity and prognosis (Figure 5, left). This trend is in contrast to the ACR guidelines appraising imaging as an inconclusive test for COVID-19 detection due to uncertainties in accuracy and risk of cross-contamination. Revealing was the unanimous use of CXR data $(50 \%$, see Figure 4, right) that was commonly utilized without any further clinical or radiomic features. The tendency for diagnosis was especially prominent for CXR versus CT where $87 \%$ and $58 \%$ diagnosis papers were found, respectively (cf. the sunburst plot showing task and maturity as distributed by modality in sup- plemental information, Figure A1). While $6 \%$ of papers (27 of all 437 non-review papers) exploited multimodal imaging data toward building their Al models, studies on multimodal imaging data of the same patient cohort are lacking with few exceptions. In one example manual disease airspace segmentation from CT was used as ground truth for volumetric quantification from CXR. ${ }^{22}$ Another study demonstrated the diagnostic accuracy of $\mathrm{Al}$ on CT to be clearly superior to CXR. ${ }^{23}$

\section{Most Al solutions for COVID-19 have low maturity}

The maturity of the papers was assessed following the scheme in Figure 1 (right) by co-authors who have developed or worked with DL algorithms (see Figure 5, middle). Almost $70 \%$ of papers were assigned a low maturity level and only $12(2.7 \%)$ highly mature studies were identified. A detailed spreadsheet with the evaluations of each paper is included in supplemental information.

CT papers had a higher maturity score than CXR papers $(2.1 \pm$ 1.3 versus $1.3 \pm 1.1, p<1 \times 10^{-11}$, Mann-Whitney $U$ test) and $57 \%$ of CT versus $43 \%$ of CXR papers were peer-reviewed. As the pandemic continues the preprint ratio is declining steadily (from $69 \%$ in Q1 to $45 \%$ in Q4) but not (yet) significantly ( $r=$ $-0.93, p=0.07$ ). The maturity score also heavily varies across performed task and was significantly higher for COVID-19 severity assessment and prognosis (2.4 and 2.5) compared with diagnosis/detection (1.5) and segmentation (1.6) as assessed by Tukey's post hoc HSD multiple comparison tests (Figure 6).

A posteriori, we observed that the monthly citation rate was significantly greater for (1) high compared with medium maturity papers (6.9 versus 2.3, $\mathrm{p}<0.01, \mathrm{U}$ test) and (2) medium compared with low maturity (2.3 versus $1.9, p<0.05, U$ test). The continuous maturity score was found to be significantly correlated $(r=0.12, p<0.05)$ with the monthly citation rate. Interestingly however, a major factor accounting for a high citation rate is not the maturity but the months elapsed since publication $\left(r=0.35, p<1 \times 10^{-14}\right)$. This suggests that absolute citations and relative citation rates are insufficient quality measures, and we instead observe a tendency toward continuous citation of publications that appeared early in the pandemic (irrespective of their quality). 


\section{Number of papers in 2019}

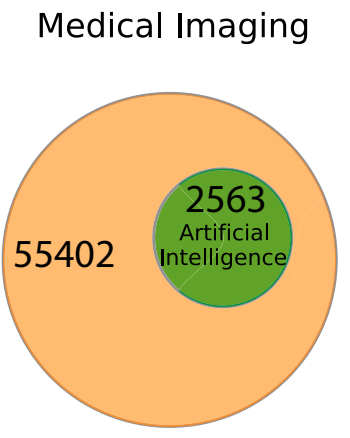

\section{Number of papers in 2020}

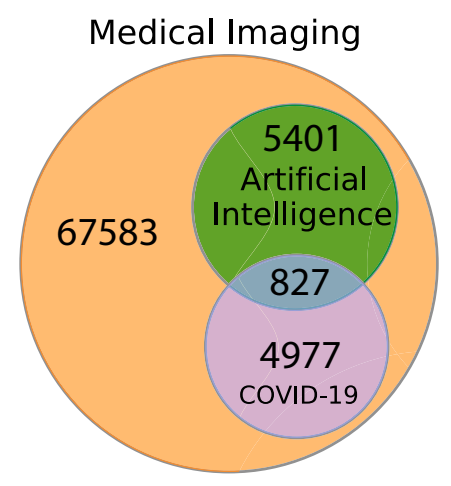

Figure 2. Venn diagrams for $\mathrm{Al}$ in $\mathrm{MI}$ MI received growing attention in 2020, at least partially due to the COVID-19 pandemic. Automatic keyword searches on PubMed and preprint servers revealed that $\mathrm{Al}$ has been a majorly growing subfield of $\mathrm{Ml}$ and that 827 publications in 2020 mentioned the terms MI, Al, and COVID-19.

2. Data sources. The majority of mature publications utilized data obtained from multiple hospitals containing imaging data from about 500-5,000 patients. The datasets were typically labeled using manual annotations from radiologists, RT-PCR tests, and results from radiology reports. Note that only

Overuse of small incomprehensive public datasets We observed that only $30 \%$ of papers used proprietary or clinical data (Figure 5, right) while almost $70 \%$ analyzed publicly available databases. Such databases exist for $\mathrm{CT},{ }^{24} \mathrm{CXR},{ }^{25}$ and LUS $^{26}$ and are usually assembled by Al researchers, contain data fetched from publications, and comprise no more than a few hundred patients from heterogeneous sources/devices without detailed patient information. Accordingly, the geographical diversity of data sources was not extremely high (26 countries), and by a wide margin the three most important data donators were countries hit early by the pandemic, namely, China (48\%) and, to a lesser extent, the United States (12\%) and Italy (11\%). Interestingly, a global collaborative spirit toward combating COVID-19 was revealed as first-authors from 53 countries and 6 continents contributed to the research, with the most active countries being China (21\%), the United States (13\%), and India (11\%).

\section{Uncovering trends in Al solutions from the mature papers}

Twelve (2.7\%) of the assessed papers were assigned high maturity. ${ }^{23,27-37}$ The list of papers together with details about their task, key findings, implementation, and results appear in Table 1 and are further discussed in this section.

We summarize the trends observed in the identified list of mature papers with a deeper focus on aspects such as (1) choice of Al model architecture, (2) diversity in data sources, (3) choice of evaluation metrics, (4) model generalization, and (5) reproducibility. Furthermore, we highlight common limitations reported in these papers.

1. Al modeling. Most of the presented Al solutions have high complexity comprising of multiple modeling stages with at least two models and at most an ensemble of 20 models $^{35}$ being trained. Solutions for segmentation tasks tend to model three-dimensional (3D) data, while classification tasks used two-dimensional (2D) data. Almost all of the solutions used transfer learning with pretraining on ImageNet or other open-source clinical datasets (e.g., CheXpert, COPDGene). Popular neural network architectures used included UNet, ResNet, DenseNet, and InceptionNet. three studies utilized clinical metadata in addition to images to develop their Al system. ${ }^{27-29}$

3. Evaluation metrics. The publications addressing diagnosis tasks commonly used metrics such as accuracy, AUC, sensitivity, and specificity to evaluate the model performance, while using Dice and intersection over union scores to quantify performance on segmentation tasks. The Pearson correlation coefficient was routinely used to compare model and human reader performances and understand the influence of learned features on the overall system performance.

4. Experimental rigor and model generalization. We observed that while most publications reported confidence intervals and performed statistical tests, they evaluated their algorithm typically only on a single random split of the dataset. Most mature publications reported model performance on external test datasets, as well as presented heatmaps to illustrate regions of image the model focused on. However, few conducted cross-validation and ablation studies to understand the generalization capabilities of their models. Furthermore, a couple of solutions were deployed in clinical practice ${ }^{31,36}$ while another was also thoroughly tested in multiple countries. ${ }^{27}$

5. Reproducibility. All of the mature publications used a human-in-the-loop (about 1-8 experienced radiologists) to compare and evaluate their proposed Al solutions, thus making such an evaluation scheme a standard practice. Moreover, a majority of the studies released the code for their algorithm publicly, while the data usually remained proprietary, but was at least partly released in four mature papers. $^{27,29,30,32}$

6. Limitations. All publications acknowledge limitations in their studies owing to inherent biases that are modeled into in the datasets through limited size, lack of diversity, and imbalance in disease conditions. In many situations, the datasets represented population of patients with higher prevalence of COVID-19 at the time of imaging, which does not reflect true disease prevalence. Furthermore, the models were deemed sensitive to motion artifacts and other subtypes of lesions or comorbidities that cause data distribution shifts. Most studies also utilized datasets from limited geographical locations, thereby 

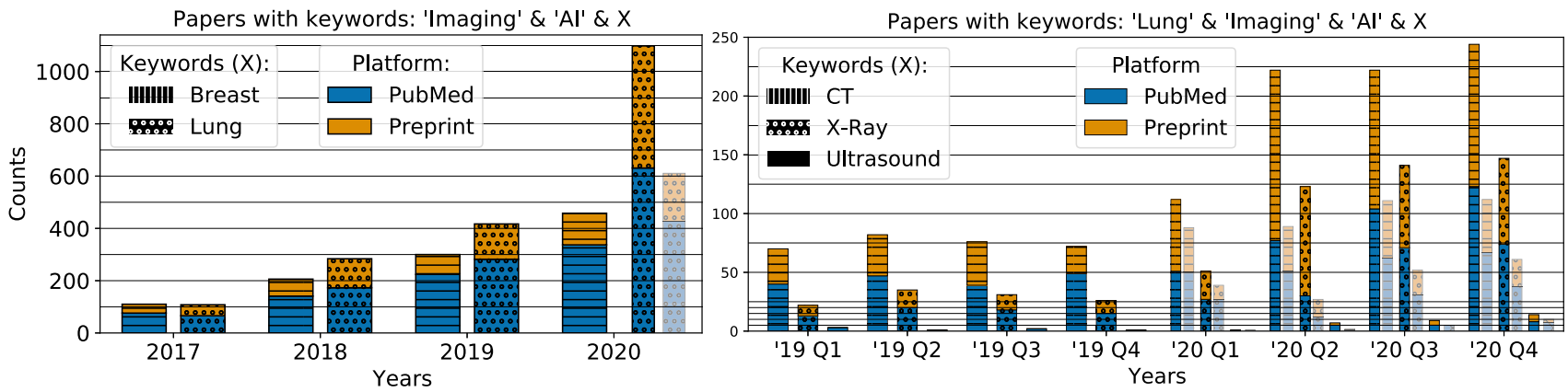

Figure 3. Number of papers per keyword and platform

Left: paper counts using Al on breast or lung imaging. At half-year resolution, the trends persisted; $a>100 \%$ growth rate for lung was visible in the first half ( $\mathrm{H} 1$ ) of 2020 whereas $\mathrm{H} 2$ brought about an additional growth of approximately one-third (not shown). The lightly shaded bars exclude COVID-19-related papers, which show the continuity of publications without COVID-19. Right: paper counts comparing the usage of Al on lung imaging modalities. COVID-19 is accompanied by a shift toward more CXR compared with CT papers. For each keyword, multiple synonyms were used (for details see appendix Table A1).

restricting generalization performance of the models in other geographies.

\section{Task-specific review of publications}

In this section, we discuss the four categories of tasks addressed by the 463 papers chosen for meta-analysis, namely diagnosis, severity, prognosis, and segmentation. We also highlight key results from the 12 mature publications and provide an overview of the findings specific to COVID-19.

\section{Diagnosis}

We find that $72 \%$ of the papers centered on COVID-19 diagnosis with 8 out of the 12 mature papers (75\%) also addressing this task. As the most prominent COVID-19 test relies on the identification of viral RNA using RT-PCR, ${ }^{38}$ imaging is not routinely performed/recommended for diagnosis and, given its reliance on pulmonary pathologies, it is especially inappropriate for detection of early or asymptomatic infections. ${ }^{39}$ However, compared with nucleic acid tests, CT may be more sensitive at a single time point for the diagnosis of COVID-19. ${ }^{40}$ A key diagnostic challenge is the non-specificity of COVID-19 patterns and their differentiation from non-COVID-19 viral pneumonia. ${ }^{41}$ Here, non-imaging assessments such as anamnesis can contribute to the diagnosis. Second, asymptomatic patients with unaffected lungs are notoriously challenging to be detected. In both cases, however, the lack of visibly distinguishing features for COVID-19 might not directly imply a limited ability of DL-based approaches, which might still be able to automatically identify (segment) distinguishing features, given the appropriate data for training. ${ }^{42}$

As has been demonstrated, if DL approaches combine CT and clinical features, the performance of radiologists in the detection of symptomatic COVID-19 patients can be matched ${ }^{28}$ (or surpassed $^{33}$ ), and even asymptomatic patients with normal CT scans can be identified in $68 \%$ of the cases. ${ }^{28}$

Moreover, multiple studies validated that radiologists' performance improves upon consultation of Al: Junior radiologists along with $\mathrm{Al}$ can perform as well as mid-senior radiologists, ${ }^{27}$ and radiologists' sensitivity and specificity can improve by nearly $10 \%$ through $\mathrm{Al}^{43}$

In another study, Al recovered full-dose CT from ultra-lowdose CTs with a satisfying acceptance score of 4.4 out of 5 by radiologists (compared with 4.7 and 2.8 for full- and ultra-lowdose, respectively) and thus helped to reduce the CT radiation dose by up to $89 \%$ while still facilitating downstream diagnosis. ${ }^{44}$ One highly mature diagnostic study using CXR included almost 6,000 scans from $>2,000$ COVID-19 patients, and their DL model exceeded the diagnostic performance of thoracic radiologists as found by significantly higher AUC of 0.94 (versus 0.85 ) and sensitivities when matching specificity to radiologists' performance. $^{35}$

\section{Severity assessment}

Imaging findings of COVID-19 patients correlate with disease severity, ${ }^{45}$ and CT scanning can assess the severity of COVID19 and help monitor disease transformation among different clinical conditions. ${ }^{46}$ A retrospective comparison of imaging findings on chest CTs with disease severity revealed an increased occurrence of consolidation, linear opacities, crazy-paving pattern, and bronchial wall thickening in severe patients at a higher frequency than in non-severe COVID-19 patients. The CT findings correlated with several worse symptoms, including a respiratory rate greater than 30 breaths per minute and oxygen saturation of $93 \%$ or less in a resting state, among other phenotypes. ${ }^{47}$ In clinical practice, often progress assessments as well as patient management is performed based on CXR and not chest CT. Al that provides assessment of severity could be useful if it was quantifiable and accurate, but only one publication was found to be mature in performing this task. ${ }^{36}$ The authors developed a clinically useful Al tool consisting of a UNet backbone for lung segmentation and quantification of pulmonary opacity within 10 days and achieved human-level performance when training on less than $200 \mathrm{CT}$ scans. ${ }^{36}$ Another work utilized a dataset of multiple CT scans per patients and introduced a "CT scan simulator" that modeled the temporal evolution of the CT through disease progression and was evaluated on multinational and multimachine data. ${ }^{48}$ Their work proposed to decompose the task of CT segmentation from one 3D into three 2D problems, thus achieving remarkable performance. Notably, despite the overall overhead of CXR compared with CT in the analyzed publications, only $3 \%(n=6)$ of the CXR publications in the metaanalysis focused on severity assessment (cf. 14\% for CT). One of them trained DL models on lung segmentation and opacity detection of 48 COVID-19 patients and achieved an agreement measure (Cohen's kappa) of 0.51 for alveolar opacities and 


\section{Clinical papers $(\mathrm{N}=2496)$}

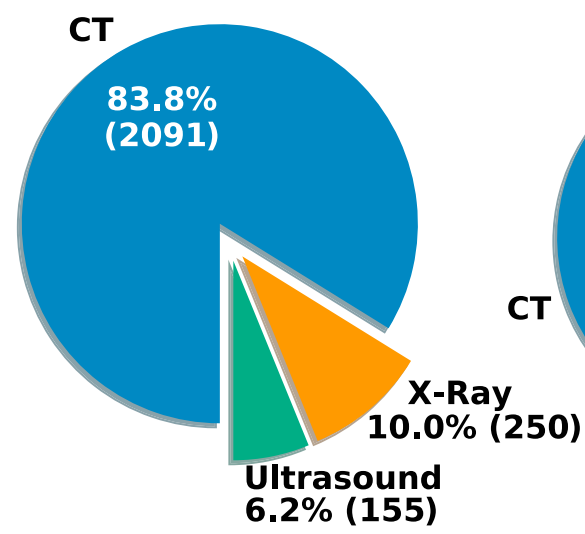

Al Papers $(\mathrm{N}=463)$

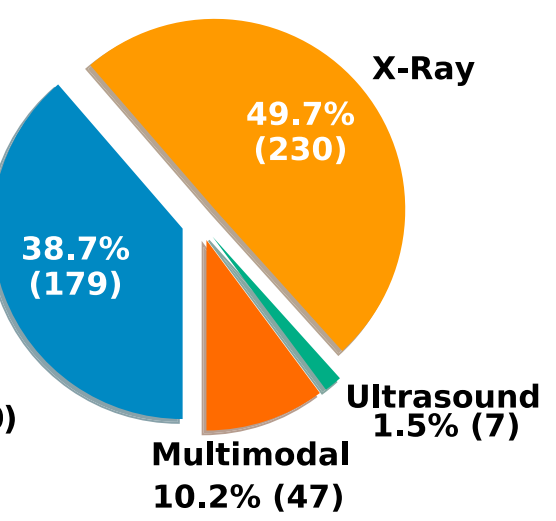

Figure 4. Imaging modality comparison during the COVID-19 pandemic

CT takes the lion's share of clinical papers about lung imaging of COVID-19 (left). The Al community (right) instead published disproportionately more papers on CXR compared with clinicians, whereas CT and ultrasound are under-represented. Multimodal papers used more than one imaging modality.

set of 3,777 patients as well as the source code is available to the public to support the development of a better system and to validate their study.

Segmentation

The main abnormalities observed in common and severe COVID-19 cases are ground-glass opacities (GGOs) and

0.71 for interstitial opacities. ${ }^{49}$ In one publication with multimodal imaging data for one patient cohort, manual airspace disease segmentation of CTs in 86 COVID-19 patients was used as ground truth to train a super-resolution convolutional neural network on volumetric quantification from CXR. The obtained correlation percentage of opacity $(\mathrm{PO})$ volume $(\mathrm{CT})$ and $\mathrm{PO}$ area (CXR) was around 0.8 for both $\mathrm{Al}$ and averaged human experts. A recent study on LUS first inferred a patient-level representation from the region-level LUS videos using attentionbased multiple-instance learning and then performed semi-supervised contrastive learning to integrate imaging with clinical data. ${ }^{50}$ The method achieved $75 \%$ and $88 \%$ accuracy in a $4-$ level/2-level patient severity assessment, respectively, and even identified infected regions in LUS (B-lines) en passant.

Prognosis

Very few of the papers (26, i.e., 6\%) focused on prognostic assessments of COVID-19 such as treatment outcome prediction, risk assessment (e.g., requirement for intensive care unit admission or mechanical ventilation), or time elapsed to negative PCR. However, two of them were assessed as mature, ${ }^{27,29}$ and the average maturity score was the highest for this task (cf. Figure 6).

However, in contrast to diagnosis, these tasks are clinically more relevant as they cannot be performed routinely and reliably with standard care. While this can be attributed to an overall gap in knowledge of the long-term effects of COVID-19 and a lack of historical data to enable training on large-scale prognosis data, it is constructive toward the alignment of future research in the field. On the other hand, in the past few months the hyper-inflammatory response induced by COVID-19 has been identified as a major cause of disease severity and death. ${ }^{51}$ Thus, studies have focused on the identification of predictive biomarkers of pathogenic inflammation. Lung imaging is not expected to reflect these biomarkers' expression, leading to limited prognosis accuracy based on imaging. One study assessed as highly mature seamlessly integrated a diagnostic module (based on a CT lung-lesion segmentation) with a prognostic module that combined clinical metadata and quantification of lung-lesion features. $^{27}$ The system demonstrated diagnostic performance comparable with a senior radiologist, and the prognostic module predicted progression to critical illness and could evaluate drug treatment efficacy by three drugs. Notably, the multicenter data- patchy consolidation surrounded by GGOs. COVID-19 pneumonia manifests with chest CT imaging abnormalities, even in asymptomatic patients, with rapid evolution from focal unilateral to diffuse bilateral GGOs that progress to or co-exist with consolidations within 1-3 weeks. ${ }^{52}$ The visual features of GGOs and consolidation lend themselves to image analysis by DL networks, and with 27 publications (8\%) segmentation became the second-most-performed task after diagnosis. In our analysis, many of the papers performed segmentation to enable other clinical tasks as discussed above, but one mature study focused on providing pulmonary lobe segmentation with relational modeling. ${ }^{30}$ Using topological modeling techniques that explore structural relationships between vessels, airways, and the pleural wall, and break up with the common strategy of utilizing fully local modules such as convolutions, they achieved human-level performance. In most cases (82\%), segmentation publications utilized external data sources with little or no clinical collaboration. Some segmentation-based models output pixelwise-labeled tissue maps of GGO or consolidation regions, providing quantitative localization of findings and identification of disease features, which can be especially informative in clinical tasks such as grading disease severity or tracking progression over time. Chaganti et al. achieved this by segmenting anatomical landmarks with reinforcement learning and computing the $\mathrm{PO}$ and lung severity score as complementary severity measures. ${ }^{53}$

In an exhaustive empirical evaluation of DL models on a clinical dataset of almost 100 COVID-19 patients, distinguishing lesion types was found more difficult than lung segmentation or binary lesion segmentation while model ensembles demonstrated best performance. ${ }^{54}$ The manual delineation from radiologists, valuable for segmentation tasks, inherently introduces some interrater variability, which underlines the need for segmentation techniques that can deal with uncertainty in annotations. ${ }^{55}$

\section{DISCUSSION}

In summary, the number of papers on $\mathrm{Al}$ in MI for COVID-19 has grown exponentially in 2020 , and the quality of the manuscripts varies significantly. In our manual review, only 12 (2.7\%) highly mature studies were identified. A key characteristic that underpins 
A

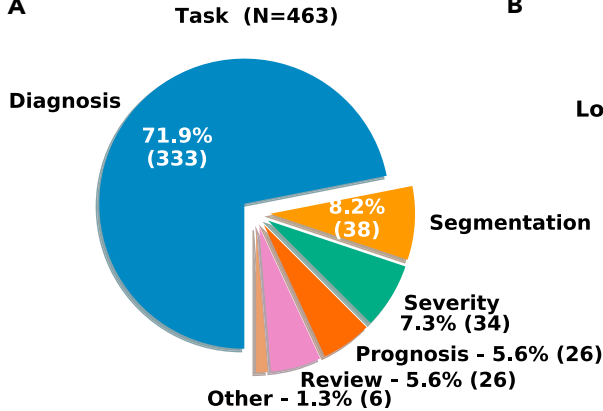

Maturity level $(\mathrm{N}=\mathbf{4 3 7}) \quad \mathrm{C}$

C

Data origin $(\mathrm{N}=437)$

Figure 5. Distribution of manually reviewed papers on Al and MI during the COVID-19 pandemic

Relative proportions for primary performed task (A), quality $(B)$, and data origin $(C)$ are given. $N$ is smaller for $(B)$ and $(C)$, since review papers were excluded from that analysis.

highly mature studies is an interdisciplinary and often multinational collaboration of medical professionals and computer vision researchers.

\section{Challenges and possible solutions}

Given the observed disparities between the Al and medical communities, we discuss several challenges that are currently encountered in such interdisciplinary collaborations and provide potential approaches to remedy the same.

\section{Choosing the right task for Al models}

The Al literature primarily addresses diagnostic tasks as opposed to other tasks with higher clinical relevance, such as monitoring/severity estimation (which tracks with clinical outcomes) and management tasks such as ventilation equipment and bed allocation. Currently even the best Al solutions have minimal performance gains on well-defined tasks (such as diagnosis) and are thus unlikely to be adopted clinically. ${ }^{8}$

Conclusions from our meta-analysis are that (1) the choice of task is critically driven by the availability of annotated data and (2) the speed of execution in Al propels blind response to increase short-term rewards instead of finding solutions to highpriority problems. This partly explains the overattention to diagnostic tasks. Moreover, classification is the canonical machinelearning $(\mathrm{ML})$ formulation and, while regression techniques can estimate non-binary severity scores, they are less frequently used. Severity estimation can be reduced to summing a classification problem on the pixel level, but this requires very expensive pixelwise annotated training data. Another common misalignment between communities is the disparate objective functions in diagnostic classification of COVID-19 from imaging data. Irrespective of the availability of direct tests for SARS-CoV-2, radiologists around the globe are steered by the objective to avoid false negatives; their decisions are less factious and dichotomous and more granular than a categorical classification of an ML model. On the other hand, the utility of an Al model, trained on a ground truth assigned by radiologists' interpretation, is limited and mostly restricted toward saving time and resources rather than getting better decisions.

To remedy and develop better clinical prediction models, the seven steps for development and four steps for validation proposed by Steyerberg et al. ${ }^{56}$ should be followed and complemented by an increased motivation among Al experts to focus on the correct questions and leverage-suitable and radiologist- friendly inductive biases such as soft labeling. ${ }^{57}$ Since Al techniques are data driven, the best way to steer $\mathrm{Al}$ practice toward more COVID-19 clinical relevance is to collect CT data with annotations for severity as well as demographics data and outcomes data. Recent collaborative, multi-institution data-collection efforts such as the NHS NCCID and RSNA's RICORD datasets precisely have CT data combined with outcomes and severity, and they are sure to lead to Al approaches with more clinical impact. Al challenge competitions are a related route for channeling Al toward CT and severity estimation. MICCAl's COVID-19 lung CT lesion segmentation challenge collected a CT dataset with detailed, radiologist-labeled lesions on the pixel level. Al-based lesion segmentation can then estimate severity by counting lesion voxels. In general, the hope is that this can be applied to longitudinal studies to track COVID-19 progression and eventually be combined with demographics and hospitalization data. Two other promising and clinically relevant endeavors are (1) usage of DL for generating standardized assessment of pulmonary involvement of COVID-19 by leveraging the newly introduced COVID-19 Reporting and Data System (CORADS) ${ }^{34}$ and (2) using DL to help evaluate treatment outcomes, e.g., by assessing changes in lesion size and volume changes. ${ }^{27}$ Transparency and reproducibility

While most authors of highly mature studies released their code (indeed three papers did not release code ${ }^{29,31,37}$ ), only one-third of them released at least part of their data. This raises concerns about reproducibility and transparency of their studies, as recently argued against a Nature study on breast cancer screening ${ }^{11}$ in a "matters arising." ${ }^{58}$ Similarly, a COVID-19 mortality prediction study ${ }^{59}$ was found to be irreproducible by three independent research groups from different countries. ${ }^{60-62}$ Given the global, unprecedented public health challenge caused by COVID-19, we strongly encourage medical researchers to follow the trends toward open-source development in the field of $\mathrm{ML}$ (which was proclaimed by various luminaries 14 years ago $^{63}$ and successfully implemented in important venues). We encourage researchers to expedite a transformation toward a common practice of validating the proposed methodology and results by publishing both code and, whenever possible, anonymized medical data, especially in academic, non-commercial settings. To help foster this transformation, conference organizers and journal editors should encourage the open sharing of code and anonymized data in their call for papers and add 


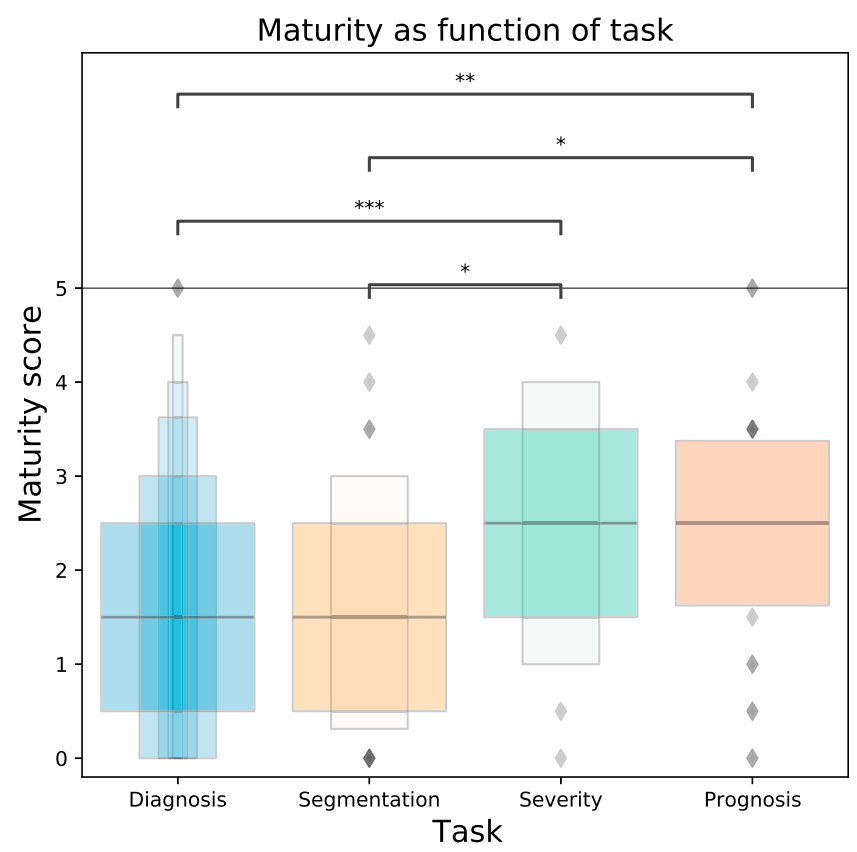

Figure 6. Maturity score as function of task $(N=437)$

Publications focusing on COVID-19 diagnosis/detection or pure segmentation achieved a significantly lower maturity score than publications addressing/ severity assessment/monitoring or prognostic tasks (asterisks indicate significance levels $0.05,0.01$, and 0.001 , respectively).

this as a criterion to the review procedure. For example, NeurIPS and ICML, premier ML conferences, expect that submissions include code and anonymized data and take this into account during the decision-making process. Similarly, the imaging conferences CVPR and MICCAI both strongly encourage the inclusion of code and data. Better guidelines from official sources such as governments are needed, especially since data-sharing regulations are less stringent during a pandemic and medical facilities are often not aware of the numerous advantages of data sharing. Privacy-preserving data science techniques have advanced $^{64}$ and should help to build more trust toward data sharing.

Federated learning (FL) is an emerging realm of ML concerned with distributed, decentralized training that stores privacy-sensitive data only locally (for details see Yang et al., Qiang and Zhang, and Vaid et al. ${ }^{65-67}$ ). FL allows multiple parties to collaboratively train the same model without data sharing and could thus become key to fostering collaborations between clinical and $\mathrm{Al}$ communities and overcoming privacy concerns. Our meta-analysis included three preprints exploring $\mathrm{FL}$ using $\mathrm{CT}^{68}$ or $\mathrm{CXR}^{69}$ data. A recent FL study on electronic health records from five hospitals was found to improve COVID-19 mortality prediction. ${ }^{70}$ These efforts will hopefully increase reproducibility and make comparative studies more feasible, which will help the research community focus on the highest-performing methods. Imaging modality rivalry

An ideal imaging modality should be safe, ubiquitous, accurate, fast, and preferably provide high-quality reproducible results via portable devices. The three different imaging modalities addressed in this study differ in their clinical use, availability, portability, safety, and reproducibility, and none of them is ideal for addressing all aspects of the pandemic (for a comparison see Table 2). For a geographic map showing the regional market sizes of the modalities, see Figure A2. Herein we have unraveled a mismatch in the number of publications per modality between clinical and Al communities: the Al literature has focused mostly on CXR whereas CT and LUS have received comparably little attention (cf. Figure 4). CT is deemed the gold standard, dominates in clinical publications, and is more sensitive than CXR for detecting diseases of the chest, but is restricted to modern medical facilities. ${ }^{71}$ CXR is notoriously less sensitive than CT, ${ }^{72}$ yet it is the most abundantly used modality across the globe when managing COVID-19 patients. While CXR can underestimate disease, CT can narrow down a differential diagnosis that appears broad on CXR. For Al, large datasets are needed for $\mathrm{ML}$ approaches, and there are much larger datasets for CXR than for CT.

As the use of imaging is less regulated compared with PCR/ antigen testing, an official recognition of all imaging modalities by leading institutions and stakeholders is needed. In conjunction with clear guidelines for clinicians on when to use which modality, trust in imaging can be increased and workflows can be streamlined. For example, the practical advantages of LUS include non-invasiveness and portability and its consequent role in triage. ${ }^{73}$ However, LUS is operator dependent and requires close patient contact for a relatively longer time. ${ }^{74}$ It was described as a preferred modality in Italy ${ }^{75}$ during spring 2020 , but it is not used as extensively in other geographic regions, being mainly applied for patients with CT/CXR contraindications and predestined to study solid organs unlike the lung. Notably, LUS sensitivity was found to be higher than that of CXR for COVID-19 diagnosis, ${ }^{76}$ and some even found comparable diagnostic accuracy to CT. ${ }^{77,78}$ However, the role of LUS for the COVID-19 pandemic is still actively debated ${ }^{79-81}$ and, regarding $\mathrm{Al}$, with only one publicly available dataset, ${ }^{26}$ more research is needed to narrow down the practical role of $\mathrm{Al}$ on LUS. ${ }^{26,50,82,83}$ Additionally, studies using ML on multiple imaging modalities from the same cohort are certainly needed to shed light on comparative questions between modalities from the perspective of ML. The performance of Al-assisted radiologists in detecting COVID-19 might or might not confirm the current radiologic findings, for example that $C X R$ is less sensitive than $\mathrm{CT}^{84}$ and LUS (when compared with RT-PCR ${ }^{76}$ or $\mathrm{CT}^{85}$ ) or that $\mathrm{B}$-lines are the most reliable pathological pattern across CT, CXR, and LUS. ${ }^{86}$ From the Al perspective, LUS is presumably the modality with the highest improvement potential in $\mathrm{Ml}$ analysis in the near future. Ultimately, Al technology focusing on plain CXR/LUS data may enable wider leverage in developing countries with limited medical resources.

\section{$M L$ interpretability}

The combined lack of robustness and interpretability poses steep challenges for the adoption of Al models in clinical practice. ${ }^{87}$ Models trained without optimizing for reliability typically make overconfident wrong predictions or underconfident correct predictions, especially when extrapolating data. To ensure that models make decisions for the right reasons, they must be trained to recognize out-of-distribution samples and handle distribution shifts, thereby allowing models to abstain from making predictions when it is unsure and deferring such samples to the experts. A human-interpretable access to the model's decision 


\begin{tabular}{|c|c|c|c|c|c|c|c|c|c|c|c|}
\hline Paper title & $\begin{array}{l}\text { Primary } \\
\text { task; } \\
\text { modality }\end{array}$ & Key findings & Limitations & $\begin{array}{l}\text { Patients } \\
\text { (train/ } \\
\text { val/test) }\end{array}$ & $\begin{array}{l}\text { No. of } \\
\text { data } \\
\text { sites }\end{array}$ & Labels & $\begin{array}{l}\text { Architecture, } \\
\text { dimensionality }\end{array}$ & Pretraining & Metrics & Results & $\begin{array}{l}\text { Reproducibility } \\
\text { (code/data } \\
\text { open source) }\end{array}$ \\
\hline $\begin{array}{l}\text { Artificial } \\
\text { intelligence- } \\
\text { enabled rapid } \\
\text { diagnosis of } \\
\text { patients with } \\
\text { COVID-19 } 19^{27}\end{array}$ & $\begin{array}{l}\text { diagnosis, } \\
\text { CT }\end{array}$ & $\begin{array}{l}\text { system } \\
\text { identified } 68 \% \\
\text { of RT-PCR- } \\
\text { positive } \\
\text { patients with } \\
\text { normal CT } \\
\text { (asymptomatic). } \\
\text { Clinical } \\
\text { information is } \\
\text { important for } \\
\text { diagnosis and } \\
\text { model is equally } \\
\text { sensitive than } \\
\text { a senior } \\
\text { radiologist }\end{array}$ & $\begin{array}{l}\text { small data } \\
\text { size, mild } \\
\text { cases have } \\
\text { few abnormal } \\
\text { findings on } \\
\text { chest CT, } \\
\text { severity of } \\
\text { pathological } \\
\text { findings } \\
\text { variable in CT }\end{array}$ & $\begin{array}{l}534 / 92 / \\
279\end{array}$ & 18 & $\begin{array}{l}\text { RT-PCR } \\
\text { tests }\end{array}$ & $\begin{array}{l}\text { Inception- } \\
\text { ResNet-v2 } \\
\text { (pretrained } \\
\text { ImageNet), } \\
\text { 3-layer } \\
\text { MLP, 2D }\end{array}$ & $\begin{array}{l}\text { transfer } \\
\text { learning } \\
\text { (pulmonary } \\
\text { tuberculosis } \\
\text { model) }\end{array}$ & $\begin{array}{l}\text { AUROC, } \\
\text { sensitivity, } \\
\text { specificity }\end{array}$ & $\begin{array}{l}0.92 \text { AUC, } \\
84.3 \% \\
\text { sens, } 82.8 \% \\
\text { spec }\end{array}$ & $\begin{array}{l}\text { code-yes, } \\
\text { data-no }\end{array}$ \\
\hline $\begin{array}{l}\text { Artificial } \\
\text { intelligence } \\
\text { augmentation } \\
\text { of radiologist } \\
\text { performance in } \\
\text { distinguishing } \\
\text { COVID-19 from } \\
\text { pneumonia of } \\
\text { other origin at } \\
\text { chest } \mathrm{CT}^{32}\end{array}$ & $\begin{array}{l}\text { Diagnosis, } \\
\text { CT }\end{array}$ & $\begin{array}{l}\text { Al assistance } \\
\text { improved } \\
\text { radiologists' } \\
\text { performance in } \\
\text { diagnosing } \\
\text { COVID-19. } \\
\text { Al alone } \\
\text { outperformed } \\
\text { radiologists on } \\
\text { sensitivity and } \\
\text { specificity }\end{array}$ & $\begin{array}{l}\text { bias in } \\
\text { radiologist- } \\
\text { annotation, } \\
\text { heterogeneous } \\
\text { data, bias in } \\
\text { location of } \\
\text { COVID (China) } \\
\text { versus non- } \\
\text { COVID } \\
\text { pneumonia } \\
\text { patients (USA) }\end{array}$ & $\begin{array}{l}830 / 237 / \\
119\end{array}$ & 13 & $\begin{array}{l}\text { RT-PCR } \\
\text { tests, slice- } \\
\text { level by } \\
\text { radiologist }\end{array}$ & $\begin{array}{l}\text { EfficientNet- } \\
\text { B4, 2D }\end{array}$ & $\begin{array}{l}\text { transfer } \\
\text { learning } \\
\text { (ImageNet) }\end{array}$ & $\begin{array}{l}\text { AUROC, } \\
\text { sensitivity, } \\
\text { specificity, } \\
\text { accuracy, } \\
\text { AUPRC }\end{array}$ & $\begin{array}{l}0.95 \text { AUC, } \\
95 \% \text { sens, } \\
96 \% \text { spec, } \\
96 \% \text { acc, } \\
0.9 \text { AUPRC }\end{array}$ & $\begin{array}{l}\text { code-yes, } \\
\text { data-no }\end{array}$ \\
\hline $\begin{array}{l}\text { Automated } \\
\text { assessment of } \\
\text { CO-RADS and } \\
\text { chest CT } \\
\text { severity scores } \\
\text { in patients with } \\
\text { suspected } \\
\text { COVID-19 } \\
\text { using artificial } \\
\text { intelligence }\end{array}$ & $\begin{array}{l}\text { diagnosis, } \\
\text { CT }\end{array}$ & $\begin{array}{l}\text { a freely } \\
\text { accessible } \\
\text { algorithm that } \\
\text { assigns CO- } \\
\text { RADS and CT } \\
\text { severity scores } \\
\text { to non-contrast } \\
\text { CT scans of } \\
\text { patients } \\
\text { suspected of } \\
\text { COVID-19 with } \\
\text { high diagnostic } \\
\text { performance }\end{array}$ & $\begin{array}{l}\text { only one data } \\
\text { center, high } \\
\text { COVID } \\
\text { prevalence, } \\
\text { low prevalence } \\
\text { for other } \\
\text { diseases }\end{array}$ & $476 / 105$ & 1 & $\begin{array}{l}\text { RT-PCR, } \\
\text { radiology } \\
\text { report }\end{array}$ & $\begin{array}{l}\text { lobe } \\
\text { segmentation } \\
\text { 3D UNet, } \\
\text { CO-RADS } \\
\text { scoring, } \\
\text { 3D Inception } \\
\text { Net }\end{array}$ & $\begin{array}{l}\text { transfer } \\
\text { learning } \\
\text { (ImageNet } \\
\text { and kinetics) }\end{array}$ & $\begin{array}{l}\text { AUC, } \\
\text { sensitivity, } \\
\text { specificity }\end{array}$ & $\begin{array}{l}\text { internal: } \\
0.95 \text { AUC, } \\
\text { external: } \\
0.88 \text { AUC }\end{array}$ & $\begin{array}{l}\text { code-yes, } \\
\text { data-no }\end{array}$ \\
\hline
\end{tabular}




\begin{tabular}{|c|c|c|c|c|c|c|c|c|c|c|c|}
\hline Paper title & $\begin{array}{l}\text { Primary } \\
\text { task; } \\
\text { modality }\end{array}$ & Key findings & Limitations & $\begin{array}{l}\text { Patients } \\
\text { (train/ } \\
\text { val/test) }\end{array}$ & $\begin{array}{l}\text { No. of } \\
\text { data } \\
\text { sites }\end{array}$ & Labels & $\begin{array}{l}\text { Architecture, } \\
\text { dimensionality }\end{array}$ & Pretraining & Metrics & Results & $\begin{array}{l}\text { Reproducibility } \\
\text { (code/data } \\
\text { open source) }\end{array}$ \\
\hline $\begin{array}{l}\text { Diagnosis of } \\
\text { Covid-19 } \\
\text { pneumonia } \\
\text { using chest } \\
\text { radiography: } \\
\text { value of } \\
\text { artificial } \\
\text { intelligence }\end{array}$ & $\begin{array}{l}\text { diagnosis, } \\
\text { X-ray }\end{array}$ & $\begin{array}{l}\text { Al surpassed } \\
\text { senior } \\
\text { radiologists in } \\
\text { COVID-19 } \\
\text { differential } \\
\text { diagnosis }\end{array}$ & $\begin{array}{l}\text { high COVID } \\
\text { prevalence, } \\
\text { human ROC- } \\
\text { AUC were } \\
\text { averaged from } \\
3 \text { readers }\end{array}$ & $\begin{array}{l}5,208 / \\
2,193\end{array}$ & $\begin{array}{l}5 \text { hospitals, } \\
30 \text { clinics }\end{array}$ & $\begin{array}{l}\text { RT-PCR, } \\
\text { natural } \\
\text { language } \\
\text { processing } \\
\text { on radiology } \\
\text { report }\end{array}$ & CV19-Net & $\begin{array}{l}\text { 3-stage } \\
\text { transfer } \\
\text { learning } \\
\text { (ImageNet) }\end{array}$ & $\begin{array}{l}\text { AUC, } \\
\text { sensitivity, } \\
\text { specificity }\end{array}$ & $\begin{array}{l}0.92 \text { AUC, } \\
88.0 \% \\
\text { sens, } 79.0 \% \\
\text { spec }\end{array}$ & $\begin{array}{l}\text { code-yes, } \\
\text { data-no }\end{array}$ \\
\hline $\begin{array}{l}\text { Development } \\
\text { and evaluation } \\
\text { of an artificial } \\
\text { intelligence } \\
\text { system for } \\
\text { COVID-19 } \\
\text { diagnosis }^{23}\end{array}$ & $\begin{array}{l}\text { diagnosis, } \\
\text { multimodal }\end{array}$ & $\begin{array}{l}\text { paired cohort } \\
\text { of chest X-ray } \\
\text { (CXR)/CT data: } \\
\text { CT is superior } \\
\text { to CXR for } \\
\text { diagnosis by } \\
\text { wide margin. } \\
\text { Al system } \\
\text { outperforms all } \\
\text { radiologists in } \\
\text { 4-class } \\
\text { classification }\end{array}$ & $\begin{array}{l}\text { more data } \\
\text { on more } \\
\text { pneumonia } \\
\text { subtypes } \\
\text { needed, no } \\
\text { clinical } \\
\text { information } \\
\text { used (could } \\
\text { enable } \\
\text { severity } \\
\text { assessment) }\end{array}$ & $\begin{array}{l}2,688 / \\
2,688 / \\
3,649\end{array}$ & 7 & - & $\begin{array}{l}\text { lung seg 2D } \\
\text { UNet, slice } \\
\text { diagnosis 2D } \\
\text { ResNet152 }\end{array}$ & $\begin{array}{l}\text { transfer } \\
\text { learning } \\
\text { (pretrained } \\
\text { ImageNet) }\end{array}$ & $\begin{array}{l}\text { AUC, } \\
\text { sensitivity, } \\
\text { specificity }\end{array}$ & AUC 0.978 & $\begin{array}{l}\text { code-yes, } \\
\text { data-no }\end{array}$ \\
\hline $\begin{array}{l}\text { Al-assisted } \\
\text { CT imaging } \\
\text { analysis for } \\
\text { COVID-19 } \\
\text { screening: } \\
\text { building and } \\
\text { deploying a } \\
\text { medical Al } \\
\text { system }^{31}\end{array}$ & $\begin{array}{l}\text { diagnosis, } \\
\text { CT }\end{array}$ & $\begin{array}{l}\text { system was } \\
\text { deployed in } \\
4 \text { weeks in } \\
16 \text { hospitals; Al } \\
\text { outperformed } \\
\text { radiologists in } \\
\text { sensitivity by } \\
\text { wide margin }\end{array}$ & $\begin{array}{l}\text { model fails } \\
\text { when multiple } \\
\text { lesions, metal } \\
\text { or motion } \\
\text { artifacts are } \\
\text { present, } \\
\text { system } \\
\text { depends } \\
\text { on fully } \\
\text { annotated }\end{array}$ & 1,136 & 5 & $\begin{array}{l}\text { Nucleic acid test, } 6 \\
\text { annotators } \\
\text { (lesions, } \\
\text { lung) }\end{array}$ & $\begin{array}{l}\text { 3D UNet++, } \\
\text { ResNet50 }\end{array}$ & full training & $\begin{array}{l}\text { sensitivity, } \\
\text { specificity }\end{array}$ & $\begin{array}{l}\text { sens } 97.4 \% \text {, } \\
\text { spec } 92.2 \%\end{array}$ & $\begin{array}{l}\text { code-no, } \\
\text { data-no }\end{array}$ \\
\hline
\end{tabular}




\begin{tabular}{|c|c|c|c|c|c|c|c|c|c|c|c|}
\hline Paper title & $\begin{array}{l}\text { Primary } \\
\text { task; } \\
\text { modality }\end{array}$ & Key findings & Limitations & $\begin{array}{l}\text { Patients } \\
\text { (train/ } \\
\text { val/test) }\end{array}$ & $\begin{array}{l}\text { No. of } \\
\text { data } \\
\text { sites }\end{array}$ & Labels & $\begin{array}{l}\text { Architecture, } \\
\text { dimensionality }\end{array}$ & Pretraining & Metrics & Results & $\begin{array}{l}\text { Reproducibility } \\
\text { (code/data } \\
\text { open source) }\end{array}$ \\
\hline $\begin{array}{l}\text { Automated } \\
\text { assessment } \\
\text { and tracking } \\
\text { of COVID-19 } \\
\text { pulmonary } \\
\text { disease } \\
\text { severity } \\
\text { on chest } \\
\text { radiographs } \\
\text { using } \\
\text { convolutional } \\
\text { Siamese neural } \\
\text { networks }\end{array}$ & $\begin{array}{l}\text { severity, } \\
\text { X-ray }\end{array}$ & $\begin{array}{l}\text { continuous } \\
\text { severity score } \\
\text { used for } \\
\text { longitudinal } \\
\text { evaluation } \\
\text { and risk } \\
\text { stratification } \\
\text { (admission } \\
\text { CXR score } \\
\text { predicts } \\
\text { intubation } \\
\text { and death, } \\
\text { AUC = 0.8). } \\
\text { Follow-up CXR } \\
\text { score by Al is } \\
\text { concordant } \\
\text { with radiologist } \\
(r=0.74)\end{array}$ & $\begin{array}{l}\text { patients only } \\
\text { from urban } \\
\text { areas in USA, no } \\
\text { generalization to } \\
\text { posteroanterior } \\
\text { radiographs }\end{array}$ & $\begin{array}{l}160,000 / \\
267 \\
\text { (images) }\end{array}$ & 2 & $\begin{array}{l}\text { RT-PCR } \\
\text { tests, 2-5 } \\
\text { annotators, } \\
\text { mRALE }\end{array}$ & $\begin{array}{l}\text { Siamese } \\
\text { DenseNet- } \\
121\end{array}$ & $\begin{array}{l}\text { DenseNet- } \\
121 \\
\text { (ImageNet, } \\
\text { fine-tuned } \\
\text { on } \\
\text { CheXpert) }\end{array}$ & $\begin{array}{l}\text { PXS score, } \\
\text { Pearson, } \\
\text { AUC }\end{array}$ & $\begin{array}{l}r=0.86 \\
A \cup C=0.8\end{array}$ & $\begin{array}{l}\text { code-yes, } \\
\text { data-partial } \\
\text { (COVID CXR } \\
\text { not released) }\end{array}$ \\
\hline $\begin{array}{l}\text { Development } \\
\text { and clinical } \\
\text { implementation } \\
\text { of tailored } \\
\text { image analysis } \\
\text { tools for } \\
\text { COVID-19 in } \\
\text { the midst of } \\
\text { the pandemic } \\
36\end{array}$ & $\begin{array}{l}\text { severity, } \\
\text { CT }\end{array}$ & $\begin{array}{l}\text { developed } \\
\text { algorithms for } \\
\text { quantification } \\
\text { of pulmonary } \\
\text { opacity in } \\
10 \text { days. } \\
\text { Human-level } \\
\text { performance } \\
\text { with <200 CT } \\
\text { scans. Model } \\
\text { integrated into } \\
\text { clinical workflow }\end{array}$ & $\begin{array}{l}\text { data: no careful } \\
\text { acquisition, not } \\
\text { complete, } \\
\text { consecutively } \\
\text { acquired or fully } \\
\text { random sample; } \\
\text { empirical HU- } \\
\text { thresholds for } \\
\text { quantification }\end{array}$ & $146 / 66$ & 1 & $\begin{array}{l}\text { RT-PCR, } 3 \\
\text { radiologist } \\
\text { annotators }\end{array}$ & 3D UNet & full training & $\begin{array}{l}\text { Dice } \\
\text { coefficient, } \\
\text { Hausdoff } \\
\text { distance }\end{array}$ & Dice $=0.97$ & $\begin{array}{l}\text { code-yes, } \\
\text { data-no }\end{array}$ \\
\hline
\end{tabular}

(Continued on next page) 


Primary

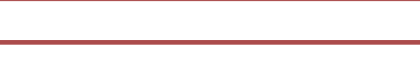

\begin{tabular}{|c|c|c|c|c|c|c|c|c|c|c|c|}
\hline Paper title & $\begin{array}{l}\text { task; } \\
\text { modality }\end{array}$ & Key findings & Limitations & $\begin{array}{l}\text { (train/ } \\
\text { val/test) }\end{array}$ & $\begin{array}{l}\text { data } \\
\text { sites }\end{array}$ & Labels & $\begin{array}{l}\text { Architecture, } \\
\text { dimensionality }\end{array}$ & Pretraining & Metrics & Results & $\begin{array}{l}\text { (code/data } \\
\text { open source }\end{array}$ \\
\hline $\begin{array}{l}\text { Clinically } \\
\text { applicable AI } \\
\text { system for } \\
\text { accurate } \\
\text { diagnosis, } \\
\text { quantitative } \\
\text { measurements, } \\
\text { and prognosis } \\
\text { of COVID-19 } \\
\text { pneumonia } \\
\text { using computed } \\
\text { tomography }{ }^{27}\end{array}$ & $\begin{array}{l}\text { prognosis, } \\
\text { CT }\end{array}$ & $\begin{array}{l}\text { Al with } \\
\text { diagnostic } \\
\text { performance } \\
\text { comparable } \\
\text { with senior } \\
\text { radiologist. Al } \\
\text { lifts junior } \\
\text { radiologists to } \\
\text { senior level. } \\
\text { Al predicts } \\
\text { drug efficacy } \\
\text { and clinical } \\
\text { prognosis. } \\
\text { Identifies } \\
\text { biomarkers } \\
\text { for novel } \\
\text { coronavirus } \\
\text { pneumonia } \\
\text { lesion. Data } \\
\text { available }\end{array}$ & & 3,777 & 4 & $\begin{array}{l}\text { pixel-level } \\
\text { annotation (5 } \\
\text { radiologists) }\end{array}$ & $\begin{array}{l}\text { lung-lesion } \\
\text { seg } \\
\text { DeepLabV3, } \\
\text { diagnosis } \\
\text { analysis 3D } \\
\text { ResNet-18, } \\
\text { gradient } \\
\text { boosting } \\
\text { decision tree }\end{array}$ & $\begin{array}{l}\text { full } \\
\text { training }\end{array}$ & $\begin{array}{l}\text { Dice } \\
\text { coefficient, } \\
\text { AUC, } \\
\text { accuracy, } \\
\text { sensitivity, } \\
\text { specificity }\end{array}$ & $\begin{array}{l}\text { AUC } 0.9797 \text {, } \\
\text { acc } 92.49 \% \text {, } \\
\text { sens } 94.93 \% \text {, } \\
\text { spec } 91.13 \%\end{array}$ & $\begin{array}{l}\text { code-yes, } \\
\text { data-yes }\end{array}$ \\
\hline $\begin{array}{l}\text { Relational } \\
\text { modeling for } \\
\text { robust and } \\
\text { efficient } \\
\text { pulmonary lobe } \\
\text { segmentation } \\
\text { in CT scans }{ }^{30}\end{array}$ & $\begin{array}{l}\text { segmentation, } \\
\text { CT }\end{array}$ & $\begin{array}{l}\text { leverages } \\
\text { structured } \\
\text { relationships } \\
\text { with non- } \\
\text { local module. } \\
\text { Can enlarge } \\
\text { receptive field } \\
\text { of convolution } \\
\text { features. } \\
\text { Robustly } \\
\text { segments } \\
\text { COVID-19 } \\
\text { infections }\end{array}$ & $\begin{array}{l}\text { errors on } \\
\text { border of } \\
\text { segmentations, } \\
\text { gross } \\
\text { pathological } \\
\text { changes not } \\
\text { represented } \\
\text { in data }\end{array}$ & $\begin{array}{l}4,370 / \\
1,100\end{array}$ & $\begin{array}{l}2 \\
\text { (pretraining: } \\
21 \text { centers) }\end{array}$ & $\begin{array}{l}\text { radiology } \\
\text { report }\end{array}$ & $\begin{array}{l}\text { RTSU-Net } \\
\text { (2-stage } \\
\text { 3D UNet) }\end{array}$ & $\begin{array}{l}\text { pretraining } \\
\text { on } \\
\text { COPDGene }\end{array}$ & $\begin{array}{l}\text { intersection } \\
\text { over union, } \\
\text { average } \\
\text { asymmetric } \\
\text { surface } \\
\text { distance }\end{array}$ & $\begin{array}{l}\text { IOU 0.953, } \\
\text { AASD } 0.541\end{array}$ & $\begin{array}{l}\text { code-yes, } \\
\text { data- } \\
\text { no/partial }\end{array}$ \\
\hline
\end{tabular}




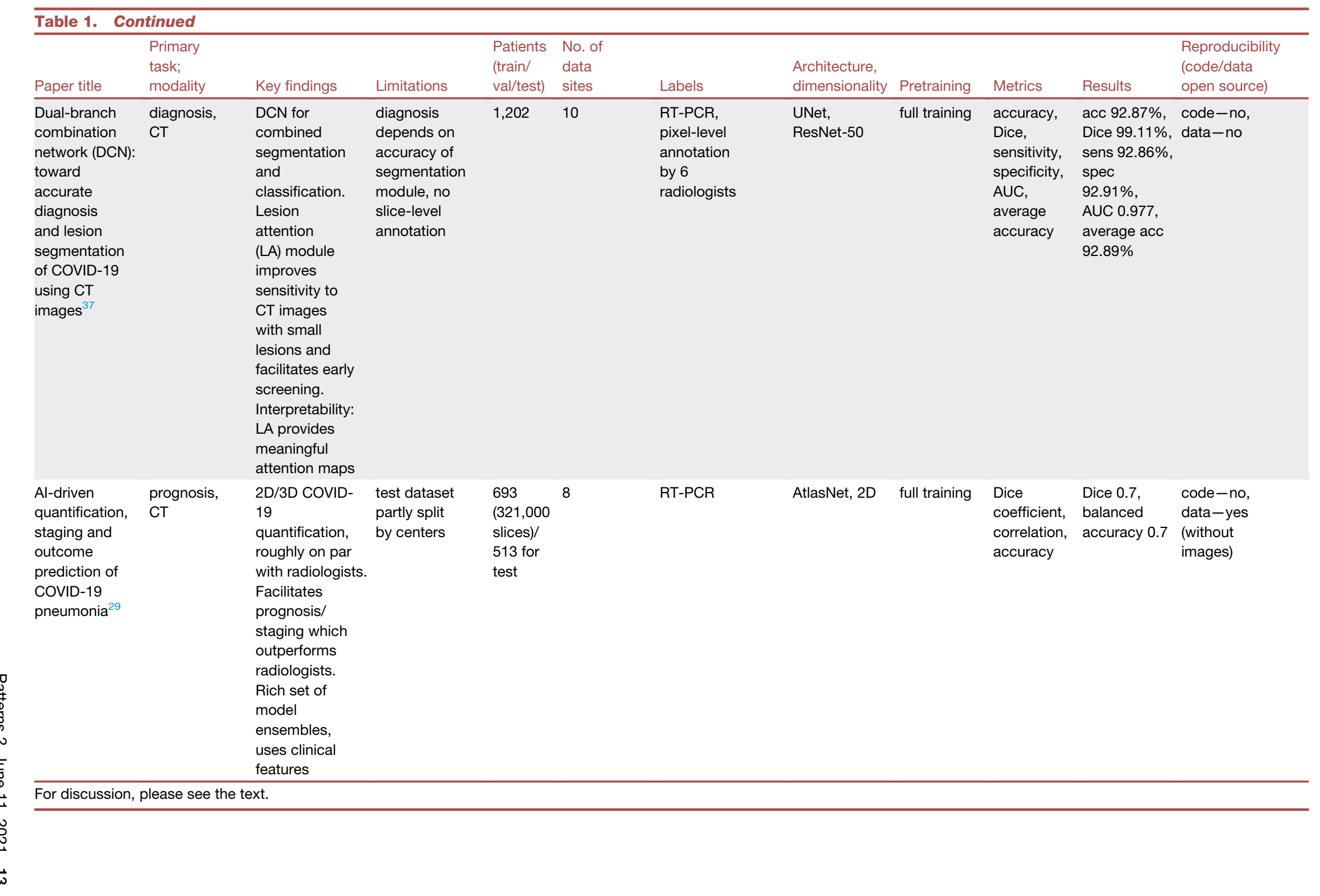




\begin{tabular}{|c|c|c|c|}
\hline \multicolumn{2}{|c|}{ CT } & CXR & US \\
\hline Benefit & $\begin{array}{l}\text { - high sensi- } \\
\text { tivity } \\
\text { - high speci- } \\
\text { ficity }\end{array}$ & $\begin{array}{l}\text { - fast } \\
\text { - broadly } \\
\text { available }\end{array}$ & $\begin{array}{l}\text { - portable } \\
\text { - radiation- } \\
\text { free } \\
\text { - broadly } \\
\text { available }\end{array}$ \\
\hline Drawback & $\begin{array}{l}\text { - patient } \\
\text { transpor- } \\
\text { tation } \\
\text { - low avail- } \\
\text { ability } \\
\text { - radiation } \\
\text { dose } \\
\text { - increased } \\
\text { workload for } \\
\text { disinfection }\end{array}$ & $\begin{array}{l}\text { - low sensi- } \\
\text { tivity } \\
\text { - non-specific } \\
\text { large volume } \\
\text { of radio- } \\
\text { graphs leads } \\
\text { to increased } \\
\text { workload }\end{array}$ & $\begin{array}{l}\text { - user- } \\
\text { dependent } \\
\text { - non-specific } \\
\text { - long acquisi- } \\
\text { tion time } \\
\text { - requires pa- } \\
\text { tient inter- } \\
\text { action }\end{array}$ \\
\hline $\begin{array}{l}\text { Clinical } \\
\text { role }\end{array}$ & $\begin{array}{l}\text { - diagnose } \\
\text { additional } \\
\text { compli- } \\
\text { cations } \\
\text { - rule out } \\
\text { additional } \\
\text { etiologies of } \\
\text { symptoms } \\
\text { (effusions, } \\
\text { bacterial } \\
\text { pneumonia) }\end{array}$ & $\begin{array}{l}\text { - initial } \\
\text { diagnosis } \\
\text { monitoring } \\
\text { clinical pro- } \\
\text { gression } \\
\text { - detection of } \\
\text { compli- } \\
\text { cations }\end{array}$ & $\begin{array}{l}\text { - triage } \\
\text { point-of-care } \\
\text { monitoring } \\
\text { for spe- } \\
\text { cific tasks }\end{array}$ \\
\hline
\end{tabular}

process is crucial to hone trust in Al, especially in medical applications where reasoning is inductive, sensitive decisions are made, and patients expect plausible hypotheses from physicians. In Ml, heatmap techniques (such as GradCAM $^{88}$ or guided-backpropagation ${ }^{89}$ ) and uncertainty estimation of individual predictions (e.g., with MC Dropout ${ }^{90,91}$ or test-timeaugmentation $^{92}$ ) are the most widely adopted approaches. However, most current interpretability tools focus on generating explanations that highlight patterns learned from the data but do not translate model decisions in human-understandable forms. Counterfactual reasoning has found its way into ML explainability ${ }^{93}$ and has opened doors toward contrastive explanations (by ascribing how changing the input would affect predictions), and can readily be combined with uncertainty quantification principles to build models integrating reliability into the optimization process. ${ }^{94}$ This will enable model introspection and facilitate human-in-the-loop analysis while also considering the performance distribution among human evaluators.

Collaboration between Al and clinical communities

A standard healthcare Al project workflow involves defining a use-case, curating data and annotations, identifying problem constraints, choosing relevant metrics, designing and building the Al system, and lastly evaluating the model performance (Figure 7, top). However, any problem involves many stakeholders: patients, ethics committees, regulatory bodies, hospital administrators, clinicians, and $\mathrm{Al}$ experts. ${ }^{95}$ In general, datadriven constraints identified by the Al experts tend to transform the clinical task into an evolved task. In combination with the disconnect of other parties (e.g., clinicians, patients) in the build life cycle, this causes potential gaps in the overall outcomes of the collaboration. Awareness and understanding of the differ- ence in needs, motivations, and solution interpretations across agents is imperative. For example, for clinicians the generation of data and metadata are cumbersome, time demanding, and tedious. What drives and motivates clinicians are improved clinical workflows and the knowledge and better understanding the analysis can bring, so that they can provide improved patient care. Moreover, Al models may hide inherent risks such as the codification of biases, the weak accountability, and the bare transparency of their decision-making process. Therefore, the way Al models are evaluated can have multiple implications on their applicability, generalization, and translation to clinical practice. ${ }^{96,96}$ To this end, both the definition of the task to be implemented and evaluated, but also the types of metrics to be leveraged to evaluate the results' outcomes, can be different across collaborators and hence must be collectively defined.

We illustrate such an improved workflow that incorporates other stakeholders in the build process, robust metrics, and iterative usability studies in Figure 7 (bottom). We believe that such a workflow could critically improve the quality of collaboration between $\mathrm{Al}$ and clinicians.

To enable agile and transparent development with continuous feedback and evaluation loops, new conducive environments are necessary. A collaboration environment that enables sharing of data, code, and results, but also immediate feedback and discussion platforms across collaborators, is essential. Communities of discovery such as the digital mammography DREAM challenge ${ }^{97}$ that bring together experts across domains under a unified cloud-based platform can enable data privacy and compliance through distributed learning and FL. Data and code sharing through open-source and open-access initiatives, and comprehensive, multidisciplinary validation could pave the way toward closing the gap between technology development and translation to clinical practice.

To summarize, the challenges toward improved collaboration include (1) aligning goals of diverse stakeholders (e.g., clinicians, Al experts, patients, funding and regulatory agencies) and (2) mapping a medical need into a well-defined task with a measurable and applicable outcome. Possible solutions include (1) inclusive execution and transparency (e.g., keep clinicians and/ or patients involved throughout the build process), (2) robust evaluation of systems (e.g., going beyond accuracy metrics to incorporate reliability metrics), and (3) creation of common work environments.

Despite the scientometric research which revealed that during COVID-19 global research investments and publication efforts have grown dramatically, ${ }^{98}$ research team sizes, number of involved countries, and ratio of international collaborations shrank. ${ }^{99}$ We therefore hope to encourage more international collaborations between the Al community and medical experts, as this could lead to more mature and conducive technologies and potentially assist clinicians and radiologists in addressing pressing clinical decision support needs during the pandemic.

\section{EXPERIMENTAL PROCEDURES}

Resource availability

Lead contact

Jannis Born (jab@zurich.ibm.com).

Materials availability

Not applicable. 


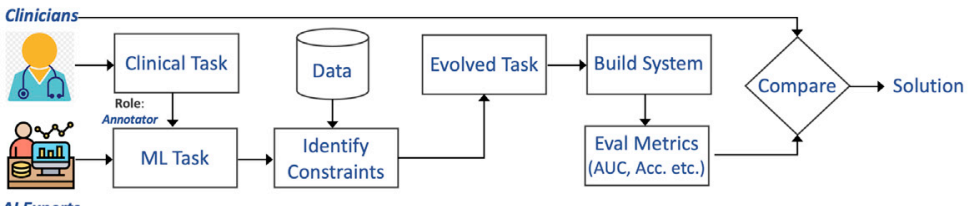

Al Experts

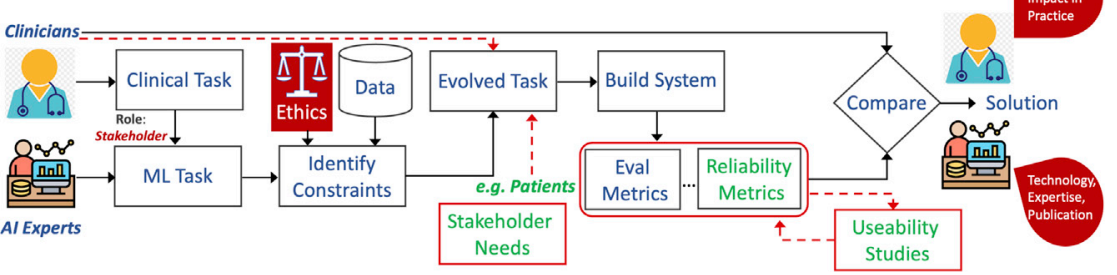

Figure 7. Workflow of collaboration between Al and clinical experts

Top: typical process of developing healthcare Al technology including task definition, data curation, building ML systems, and human-in-the-loop evaluation. Bottom: our proposed workflow, highlighting key components that need to be incorporated into the process to improve collaboration between $\mathrm{Al}$ and clinical experts. Note the disparity in value interpretation of the developed solutions by the two communities.
Data and code availability

The source code used for the publication keyword search is available via https://pypi.org/project/paperscraper/. A spreadsheet with the detailed results of the publication meta-analysis is enclosed as supplemental information (online only).

\section{Methods}

To discover trends from the overwhelming research activities in COVID-19, Al and MI, we performed a systematic review and meta-analysis according to the PRISMA guidelines. ${ }^{100}$ Literature, indexed in PubMed and three preprint servers, namely arXiv, bioRxiv, and medRxiv, were queried. The process is illustrated in Figure 1 (left) and shows two main streams of queries: a broad one using "Al" AND "COVID-19" AND "Medical Imaging" and a modality-specific one with "Al" AND "COVID-19" AND "Lung" AND ("CT" OR "CXR" OR "US"). Following PRISMA guidelines, we combined the results of both queries across all databases leading to the identification of 463 papers about $\mathrm{Al}$ on lung imaging for COVID-19. These papers were included in a manual meta-analysis to review the maturity of the $\mathrm{Al}$ technologies and the trends in the rapidly evolving field (for the detailed procedure and a list of synonyms used, see appendix Table A1). The publications about Al technology typically tend to report a proof of concept, an illustration of a success in a non-clinical setting, or a report of clinically successful experiments. Additionally, many of the papers identified were not published in peer-reviewed journals. To evaluate the maturity of papers, we included five criteria that were assessed rigorously (Figure 1, right).

1. Peer review: Whether or not the paper appeared in a peer-reviewed journal or conference.

2. Modeling quality: The complexity and the performance of the developed Al framework.

3. Data quality/scale: Number of patients in the data used for training and evaluation. Internal, clinical data is preferred over public datasets, and multihospital/multimodal data are valued.

4. Experimental rigor: Stringency in the evaluation and comparison of the methodology.

5. Clinical deployment: The deployment and adoption of the solution in hospitals. Comparison studies of $\mathrm{Al}$ and radiologists or deployment of web services were also rewarded.

The peer-review score was binary and all other categories were scored ternarily $(0,0.5,1)$. Details of the scheme with examples can be found in the supplemental information. The decision function for maturity level (Figure 1, right) guarantees that publications which received a " 0 " in one of the five categories cannot obtain a high maturity score (implying that, e.g., preprints are never highly mature).

Moreover, we manually inferred the most common tasks addressed in the $\mathrm{Al}$ papers, such as detection, segmentation, characterization, and outcome prediction, and mapped them into three main clinically relevant categories-diagnosis, severity assessment, and prognosis - and one technical task, segmentation. The segmentation papers discuss localization of lung tissue or other disease features without direct applications to any clinically relevant downstream tasks.

For publications that focused on several categories, we consider the primary task only. For example, a number of publications classified as "diagnosis" or "severity assessment" utilized segmentation methods on the fly. Papers that provided a review of ML for MI on COVID-19 and did not introduce original new technology were labeled as "review" papers and excluded from the maturity assessment, leading to 437 reviewed papers. The remaining evaluation criteria per publication were imaging modality, country of authors, and country of data source. For each paper, we also recorded the total number of citations indicated on Google Scholar as of February 28, .2021 and converted it to the monthly citation rate. Note that the meta-analysis was blindfolded to the number of citations.

The publication keyword search was performed using our toolbox paperscraper that was developed during this project and is open-sourced (https:// pypi.org/project/paperscraper/).

\section{SUPPLEMENTAL INFORMATION}

Supplemental information can be found online at https://doi.org/10.1016/j patter.2021.100269.

\section{AUTHOR CONTRIBUTIONS}

Conceptualization, M.R.-Z., D. Beymer, J.L.R., E.K., M. Gabrani, J.B., and D.R.; Methodology, M.R.-Z., M. Gabrani, D. Beymer, J.B., and D.R.; Software, J.B. and M.M.; Validation, M.R.-Z., M. Gabrani, D. Beymer, and J.B.; Formal analysis, J.B. and D.R.; Investigation, J.B., D.R., D. Beymer, A.C., V.V.M., E.K., and M. Gabrani; Data curation, J.B.; Writing - original draft, J.B., D. Beymer, D.R., M. Gabrani, M.R.-Z., J.L.R., E.K., A.C., P.L.S., D.S., and M. Guindy; Writing - review \& editing, J.B., D.R., M. Gabrani, D. Beymer, and M.R.-Z.; Visualization, J.B., D.R., and M. Gabrani; Supervision, M.R.-Z., M. Gabrani, J.L.R., E.K., P.L.S., A.C., D.S., M. Guindy, V.V.M., P.P., and D. Ballah; Project administration, M.R.-Z., M. Gabrani, and D. Beymer.

\section{DECLARATION OF INTERESTS}

The authors declare no competing interests.

\section{REFERENCES}

1. Dong, D., et al. (2020). The role of imaging in the detection and management of COVID-19: a review. IEEE Rev. Biomed. Eng. 14, 16-29. https:// doi.org/10.1109/RBME.2020.2990959.

2. Pascarella, G., et al. (2020). "COVID-19 diagnosis and management: a comprehensive review. J. Intern. Med. 288, 192-206.

3. Rubin, G.D., Ryerson, C.J., Haramati, L.B., Sverzellati, N., Kanne, J.P Raoof, S., et al. (2020). The role of chest imaging in patient management during the COVID-19 pandemic: a multinational consensus statement from the Fleischner Society. Chest 158, 106-116. https://doi.org/10. 1016/j.chest.2020.04.003.

4. American College of Radiology (2020). ACR recommendations for the use of chest radiography and computed tomography (CT) for suspected COVID-19 infection, Updated March, 22 https://www.acr.org/Advocacyand-Economics/ACR-Position-Statements/Recommendations-for-ChestRadiography-and-CT-for-Suspected-COVID19-Infection. 
5. Lomoro, P., et al. (2020). COVID-19 pneumonia manifestations at the admission on chest ultrasound, radiographs, and CT: single-center study and comprehensive radiologic literature review. Eur. J. Radiol. Open 7, 100231. https://doi.org/10.1016/j.ejro.2020.100231.

6. Shi, F., Wang, J., Shi, J., Wu, Z., Wang, Q., Tang, Z., et al. (2020). Review of artificial intelligence techniques in imaging data acquisition, segmentation and diagnosis for Covid-19. IEEE Rev. Biomed. Eng. 14, 4-15. https://doi.org/10.1109/RBME.2020.2987975.

7. L. Wynants et al., "Prediction models for diagnosis and prognosis of Covid-19: systematic review and critical appraisal," BMJ, vol. 369, p. 18, Apr. 20 .

8. Celi, L.A., Fine, B., and Stone, D.J. (2019). An awakening in medicine: the partnership of humanity and intelligent machines. Lancet Digit. Health 1, e255-e257.

9. Kim, H.E., et al. (2020). Changes in cancer detection and false-positive recall in mammography using artificial intelligence: a retrospective, multireader study. Lancet Digit. Heal. 2, e138-e148.

10. Akselrod-Ballin, A., et al. (2019). Predicting breast cancer by applying deep learning to linked health records and mammograms. Radiology 292, 331-342.

11. McKinney, S.M., et al. (2020). International evaluation of an Al system for breast cancer screening. Nature 577, 89-94.

12. Le, E.P.V., Wang, Y., Huang, Y., Hickman, S., and Gilbert, F.J. (2019). Artificial intelligence in breast imaging. . Clinical Radiology, 74 (W.B. Saunders Ltd), pp. 357-366.

13. Halling-Brown, M.D., et al. (2020). OPTIMAM mammography image database: a large-scale resource of mammography images and clinical data. Radiol. Artif. Intell. e200103.

14. Irvin, J., et al. (2019). CheXpert: a large chest radiograph dataset with uncertainty labels and expert comparison. Proc. AAAl Conf. Artif. Intell. 33, 590-597.

15. Ardila, D., et al. (2019). End-to-end lung cancer screening with threedimensional deep learning on low-dose chest computed tomography. Nat. Med. 25, 954-961.

16. Saba, L., Biswas, M., Kuppili, V., Godia, E.C., Suri, H.S., Edla, D.R., Omerzu, T., Laird, J.R., Khanna, N.N., Mavrogeni, S., and Protogerou, A. (2019). The present and future of deep learning in radiology. Eur. J. Radiol. 114, 14-24.

17. Syeda, H.B., et al. (2021). Role of machine learning techniques to tackle the COVID-19 crisis: systematic review. JMIR Med. Inform. 9, e23811.

18. Chiroma, H., et al. (2020). Early survey with bibliometric analysis on machine learning approaches in controlling COVID-19 outbreaks. PeerJ Computer Sci. 6, e313.

19. Albahri, O.S., et al. (2020). Systematic review of artificial intelligence techniques in the detection and classification of COVID-19 medical images in terms of evaluation and benchmarking: Taxonomy analysis, challenges, future solutions and methodological aspects. J. Infect. Public Health 13, 1381-1396. https://doi.org/10.1016/j.jiph.2020.06.028.

20. Roberts, M., et al. (2021). Common pitfalls and recommendations for using machine learning to detect and prognosticate for COVID-19 using chest radiographs and CT scans. Nat. Machine Intelligence 3, 199-217.

21. Gupta, A., et al. (2020). Extrapulmonary manifestations of COVID-19. Nat. Med. 26, 1017-1032.

22. Barbosa, E.J.M., Jr., et al. (2021). Automated detection and quantification of COVID-19 airspace disease on chest radiographs: a novel approach achieving expert radiologist-level performance using a deep convolutional neural network trained on digital reconstructed radiographs from computed tomography-derived ground truth. Invest. Radiol., In press. https://doi.org/10.1097/RLI.0000000000000763.

23. Jin, C., et al. (2020). Development and evaluation of an artificial intelligence system for COVID-19 diagnosis. Nat. Commun. 11, 5088.

24. Soares, E., et al. (2020). SARS-CoV-2 CT-scan dataset: a large dataset of real patients CT scans for SARS-CoV-2 identification. medRxiv. https:// doi.org/10.1101/2020.04.24.20078584.
25. Cohen, J.P., et al. (2020). Covid-19 image data collection: prospective predictions are the future. Journal of Machine Learning for Biomedical Imaging (MELBA), arXiv:2006.11988.

26. Born, J., Wiedemann, N., Cossio, M., Buhre, C., Brändle, G., Leidermann, K., Aujayeb, A., Moor, M., Rieck, B., and Borgwardt, K. (2021) Accelerating detection of lung pathologies with explainable ultrasound image analysis. Appl. Sci. 11, 672. https://doi.org/10.3390/ app11020672.

27. Zhang, K., Liu, X., Shen, J., Li, Z., Sang, Y., Wu, X., et al. (2020). Clinically applicable Al system for accurate diagnosis, quantitative measurements, and prognosis of COVID-19 pneumonia using computed tomography. Cell 181,1360 .

28. Mei, X., Lee, H.-C., Diao, K.-Y., Huang, M., Lin, B., Liu, C., et al. (2020). Artificial intelligence-enabled rapid diagnosis of patients with COVID19. Nat. Med. 26, 1224-1228.

29. Chassagnon, G., et al. (2020). Al-driven quantification, staging and outcome prediction of COVID-19 pneumonia. Med. Image Anal. 67, 101860.

30. Xie, W., et al. (2020). Relational modeling for robust and efficient pulmonary lobe segmentation in CT scans. IEEE Trans. Med. Imaging 39 2664-2675.

31. Wang, B., et al. (2020). Al-assisted CT imaging analysis for COVID-19 screening: building and deploying a medical Al system. Appl. Soft Comput. 98, 106897

32. Li, M.D., et al. (2020). Automated assessment and tracking of COVID-19 pulmonary disease severity on chest radiographs using convolutional Siamese neural networks. Radiol. Artif. Intelligence 2, e200079.

33. Bai, H.X., Wang, R., Xiong, Z., Hsieh, B., Chang, K., Halsey, K., et al. (2020). Artificial intelligence augmentation of radiologist performance in distinguishing COVID-19 from pneumonia of other origin at chest CT. Radiology 296. https://doi.org/10.1148/radiol.2021219004.

34. Lessmann, N., Sánchez, C.I., Beenen, L., Boulogne, L.H., Brink, M., Calli, E., et al. (2020). Automated assessment of CO-RADS and chest CT severity scores in patients with suspected COVID-19 using artificial intelligence. Radiology, 202439

35. Zhang, R., et al. (2020). Diagnosis of covid-19 pneumonia using chest radiography: value of artificial intelligence. Radiology, 202944.

36. Anastasopoulos, C., Weikert, T., Yang, S., Abdulkadir, A., Schmülling, L., Bühler, C., et al. (2020). Development and clinical implementation of tailored image analysis tools for COVID-19 in the midst of the pandemic: the synergetic effect of an open, clinically embedded software development platform and machine learning. Eur. J. Radiol. 131, 109233.

37. Gao, K., et al. (2020). Dual-branch combination network (DCN): towards accurate diagnosis and lesion segmentation of COVID-19 using CT images. Med. image Anal. 67, 101836.

38. Pascarella, G., et al. (2020). COVID-19 diagnosis and management: a comprehensive review. J. Intern. Med. 288, 192-206.

39. Tang, Y.W., Schmitz, J.E., Persing, D.H., and Stratton, C.W. (2020). Laboratory diagnosis of COVID-19: current issues and challenges. J. Clin. Microbiol. 58. https://doi.org/10.1128/JCM.00512-20.

40. Ai, T., et al. (2020). Correlation of chest CT and RT-PCR testing in coronavirus disease 2019 (COVID-19) in China: a report of 1014 cases. Radiology, 200642.

41. Mendel, J.B., Lee, J.T., and Rosman, D. (2020). Current concepts imaging in COVID-19 and the challenges for low and middle income countries. J. Glob. Radiol.

42. Li, L., et al. (2020). Artificial intelligence distinguishes Covid-19 from community acquired pneumonia on chest CT. Radiology 296, E65-E72. https://doi.org/10.1148/radiol.2020200905.

43. Song, J., et al. (2020). End-to-end automatic differentiation of the corona virus disease 2019 (COVID-19) from viral pneumonia based on chest CT. Eur. J. Nucl. Med. Mol. Imaging. 
44. Shiri, l., et al. (2020). Ultra-low-dose chest CT imaging of COVID-19 patients using a deep residual neural network. Eur. Radiol. 31, 1420-1431. https://doi.org/10.1007/s00330-020-07225-6.

45. World Health Organization, Use of chest imaging in COVID-19: a rapid advice guide, 2020.

46. Tabatabaei, S.M.H., Talari, H., Moghaddas, F., and Rajebi, H. (2020) Computed tomographic features and short-term prognosis of coronavirus disease 2019 (COVID-19) pneumonia: a single-center study from Kashan, Iran. Radiol. Cardiothorac. Imaging 2, e200130.

47. Li, M., et al. (2020). Coronavirus disease (COVID-19): spectrum of CT findings and temporal progression of the disease. Acad. Radiol. 27, 603-608.

48. Zhou, L., et al. (2020). A rapid, accurate and machine-agnostic segmentation and quantification method for CT-based COVID-19 diagnosis. IEEE Trans. Med. Imaging 39, 2638-2652.

49. Blain, M., et al. (2021). Determination of disease severity in COVID-19 patients using deep learning in chest X-ray images. Diagn. Interv. Radiol. $27,20$.

50. Xue, W., Cao, C., Liu, J., Duan, Y., Cao, H., Wang, J.,.., and Xie, M. (2021) Modality alignment contrastive learning for severity assessment of COVID-19 from lung ultrasound and clinical information. Med. Image Anal. 69, 101975.

51. Del Valle, D.M., Kim-Schulze, S., Huang, H.H., Beckmann, N.D., Nirenberg, S., Wang, B., Lavin, Y., Swartz, T.H., Madduri, D., Stock, A., and Marron, T.U. (2020). An inflammatory cytokine signature predicts COVID-19 severity and survival. Nat. Med. 26, 1636-1643.

52. Shi, H., et al. (2020). Radiological findings from 81 patients with COVID19 pneumonia in Wuhan, China: a descriptive study. Lancet Infect. Dis. 20, 425-434.

53. Chaganti, S., et al. (2020). Automated quantification of CT patterns associated with COVID-19 from chest CT. Radiol. Artif. Intelligence 2, e200048.

54. Tilborghs, S., et al. (2020). Comparative study of deep learning methods for the automatic segmentation of lung, lesion and lesion type in CT scans of COVID-19 patients. arXiv, arXiv:2007.15546.

55. Wang, G., et al. (2020). A noise-robust framework for automatic segmentation of covid-19 pneumonia lesions from ct images. IEEE Trans. Med. Imaging 39, 2653-2663.

56. Steyerberg, E.W., and Vergouwe., Y. (2014). Towards better clinical prediction models: seven steps for development and an ABCD for validation. Eur. Heart J. 35, 1925-1931.

57. Nguyen, Q., Valizadegan, H., and Hauskrecht, M. (2014). Learning classification models with soft-label information. J. Am. Med. Inform. Assoc. 21, 501-508.

58. Haibe-Kains, B., et al. (2020). Transparency and reproducibility in artificial intelligence. Nature 586, E14-E16.

59. Yan, Li, et al. (2020). An interpretable mortality prediction model for COVID-19 patients. Nat. Mach. Intell. 2, 283-288.

60. Barish, M., et al. (2021). External validation demonstrates limited clinical utility of the interpretable mortality prediction model for patients with COVID-19. Nat. Mach. Intell. 3, 25-27.

61. Quanjel, M.J.R., et al. (2020). Replication of a mortality prediction model in Dutch patients with COVID-19. Nat. Mach. Intell. 2, 23-24.

62. Dupuis, C., et al. (2020). Limited applicability of a COVID-19 specific mortality prediction rule to the intensive care setting. Nat. Mach. Intell. 2, 20-22.

63. Sonnenburg, S., et al. (2007). The need for open source software in machine learning. J. Mach. Learn. Res. 8, 2443-2466.

64. Liang, X., et al. (2017). Integrating blockchain for data sharing and collaboration in mobile healthcare applications. In IEEE 28th Annual International Symposium on Personal, Indoor, and Mobile Radio Communications (PIMRC) (IEEE). https://doi.org/10.1109/PIMRC.2017.8292361.
65. Yang, Q., et al. (2019). Federated machine learning: concept and applications. ACM Trans. Intell. Syst. Technology (Tist) 10, 1-19.

66. Qian, F., and Zhang, A. (2021). The value of federated learning during and post COVID-19. Int. J. Qual. Health Care 33, 1353-4505. https://doi.org/ 10.1093/intqhc/mzab010.

67. Vaid, A., Jaladanki, S.K., Xu, J., Teng, S., Kumar, A., Lee, S.,., and Glicksberg, B.S. (2021). Federated learning of electronic health records to improve mortality prediction in hospitalized patients with COVID-19: machine learning approach. JMIR Med. Inform. 9, e24207.

68. Kumar, R., et al. (2020). Blockchain-federated-learning and deep learning models for covid-19 detection using ct imaging. arXiv, arXiv:2007.06537.

69. Liu, B., et al. (2020). Experiments of federated learning for covid-19 chest x-ray images. arXiv, arXiv:2007.05592.

70. Vaid, A., Jaladanki, S.K., Xu, J., Teng, S., Kumar, A., Lee, S.,., and Glicksberg, B.S. (2021). Federated learning of electronic health records to improve mortality prediction in hospitalized patients with COVID-19: machine learning approach. JMIR Med. Inform. 9, e24207.

71. Castillo, M. (2012). The industry of CT scanning. Am. Soc. Neuroradiol. 33, 583-585. https://doi.org/10.3174/ajnr.A2742.

72. Wong, H.Y.F., et al. (2019). Frequency and distribution of chest radiographic findings in COVID-19 positive patients. Radiology, 201160.

73. Smith, M.J., Hayward, S.A., Innes, S.M., and Miller, A. (2020). Point-ofcare lung ultrasound in patients with COVID-19-a narrative review. Anaesthesia 75, 1096-1104.

74. Akl, E.A., Blazic, I., Yaacoub, S., Frija, G., Chou, R., Appiah, J.A., Fatehi, M., Flor, N., Hitti, E., Jafri, H., et al. (2020). Use of chest imaging in the diagnosis and management of COVID-19: a WHO rapid advice guide. Radiology, 203173. https://doi.org/10.1148/radiol.2020203173.

75. Vetrugno, L., et al. (2020). Our Italian experience using lung ultrasound for identification, grading and serial follow-up of severity of lung involvement for management of patients with COVID-19. Echocardiography 37, 625-627.

76. Pare, J.R., Camelo, I., Mayo, K.C., Leo, M.M., Dugas, J.N., Nelson, K.P., et al. (2020). Point-of-care lung ultrasound is more sensitive than chest radiograph for evaluation of COVID-19. West. J. Emerg. Med. Integr. Emerg. Care Popul. Heal. 21, 771-778. https://doi.org/10.5811/westjem.2020.5.47743.

77. Lieveld, A.W.E., et al. (2020). Diagnosing COVID-19 pneumonia in pandemic setting: lung Ultrasound versus CT (LUVCT) A multi-centre, prospective, observational study. ERJ Open Res. 6, 00539-02020. https://doi.org/10.1183/23120541.00539-2020.

78. Tung-Chen, Y., Martí de Gracia, M., Díez-Tascón, A., Alonso-González, R., Agudo-Fernández, S., Parra-Gordo, M.L., Ossaba-Vélez, S., Rodríguez-Fuertes, P., and Llamas-Fuentes, R. (2020). Correlation between chest computed tomography and lung ultrasonography in patients with coronavirus disease 2019 (COVID-19). Ultrasound Med. Biol. 46, 2918-2926.

79. Tung-Chen, Y., et al. (2020). Correlation between chest computed tomography and lung ultrasonography in patients with coronavirus disease 2019 (COVID-19). Ultrasound Med. Biol. 46, 2918-2926. https://doi.org/ 10.1016/j.ultrasmedbio.2020.07.003.

80. Buonsenso, D., Pata, D., and Chiaretti, A. (2020). COVID-19 outbreak: less stethoscope, more ultrasound. Lancet Respir. Med. 8. https://doi. org/10.1016/S2213-2600(20)30120-X.

81. Cheung, J.C.H., and Lam, K.N. (2020). POCUS in COVID-19: pearls and pitfalls. Lancet Respir. Med. 8, e34.

82. Arntfield, R., et al. (2021). Development of a convolutional neural network to differentiate among the etiology of similar appearing pathological B lines on lung ultrasound: a deep learning study. BMJ open 11, e045120.

83. Liu, L., et al. (2020). Semi-supervised active learning for COVID-19 lung ultrasound multi-symptom classification. In IEEE 32nd International Conference on Tools with Artificial Intelligence (ICTAI) (IEEE), p. 2020. 
84. Borakati, A., et al. (2020). Diagnostic accuracy of X-ray versus CT in COVID-19: a propensity-matched database study. BMJ open 10, e042946.

85. Gibbons, R.C., et al. (2020). Lung ultrasound versus chest X-ray for the diagnosis of COVID-19 pneumonia. Ann. Emerg. Med. 76, S3.

86. Lomoro, P., et al. (2020). COVID-19 pneumonia manifestations at the admission on chest ultrasound, radiographs, and CT: single-center study and comprehensive radiologic literature review. Eur. J. Radiol. open 7, 100231.

87. Reyes, M., et al. (2020). On the interpretability of artificial intelligence in radiology: challenges and opportunities. Radiol. Artif. Intell. 2, e190043.

88. Selvaraju, R.R., et al. (2017). Grad-cam: visual explanations from deep networks via gradient-based localization. Proc. IEEE Int. Conf. Comput. Vis. 618-626.

89. Springenberg, J.T., Dosovitskiy, A., Brox, T., and Riedmiller, M.A. (2015). Striving for Simplicity: The All Convolutional Net. In ICLR (Workshop).

90. Gal, Y., and Ghahramani, Z. (2016). Dropout as a bayesian approximation: representing model uncertainty in deep learning. In International Conference on Machine Learning (PMLR), pp. 1050-1059.

91. Nair, T., Precup, D., Arnold, D.L., and Arbel, T. (2020). Exploring uncertainty measures in deep networks for multiple sclerosis lesion detection and segmentation. Med. image Anal. 59, 101557.

92. Ayhan, M.S., and Berens, P. (2018). Test-time data augmentation for estimation of heteroscedastic aleatoric uncertainty in deep neural networks. In International Conference on Medical Imaging with Deep Learning. https://openreview.net/pdf?id=rJZz-knjz\%20.
93. Byrne, R.M.J. (2019). Counterfactuals in explainable artificial intelligence (XAI): evidence from human reasoning. IJCAI, 6276-6282.

94. Thiagarajan, J.J., et al. (2020). Improving reliability of clinical models us ing prediction calibration. In Uncertainty for Safe Utilization of Machine Learning in Medical Imaging, and Graphs in Biomedical Image Analysis, C.H. Sudre, H. Fehri, T. Arbel, C.F. Baumgartner, A. Dalca, R. Tanno, K. Van Leemput, W.M. Wells, A. Sotiras, and B. Papiez, et al., eds. (Springer), pp. 71-80.

95. Nagendran, M., et al. (2020). Artificial intelligence versus clinicians: systematic review of design, reporting standards, and claims of deep learning studies. BMJ, 368. https://doi.org/10.1136/bmj.m689.

96. Kelly, C.J., et al. (2019). Key challenges for delivering clinical impact with artificial intelligence. BMC Med. 17. https://doi.org/10.1186/s12916019-1426-2.

97. Schaffter, T., et al. (2020). Evaluation of combined artificial intelligence and radiologist assessment to interpret screening mammograms. JAMA Netw. open 3, e200265.

98. Organization for Economic Cooperation and Development (2021). OECD Science, Technology and Innovation Outlook 2021. https://www.oecd. org/sti/oecd-science-technology-and-innovation-outlook-25186167. htm\%20.

99. Cai, X., Fry, C.V., and Wagner, C.S. (2021). International collaboration during the COVID-19 crisis: autumn 2020 developments. Scientometrics, 3683-3692.

100. Moher, D., et al. (2009). Preferred reporting items for systematic reviews and meta-analyses: the PRISMA statement. Plos Med. 6, e1000097. 